\title{
A micro-scale inspired chemo-mechanical model of bonded geomaterials
}

\author{
Alessandro Gajo ${ }^{\mathrm{a}}$, Francesco Cecinato ${ }^{\mathrm{a}, *}$, Tomasz Hueckel ${ }^{\mathrm{b}}$ \\ ${ }^{a}$ University of Trento, Department of Civil, Environmental and Mechanical Engineering, Trento, Italy \\ ${ }^{\mathrm{b}}$ Duke University, Civil and Environmental Engineering, Durham, NC, USA
}

\section{A R T I C L E I N F O}

\section{Article history:}

Received 2 April 2015

Received in revised form

28 July 2015

Accepted 8 October 2015

\section{Keywords:}

Chemo-mechanical coupling

Constitutive modeling

Micro-macro relationships

Bonded geomaterials

Deposition-dissolution

Cementation-destructuration

\begin{abstract}
A B S T R A C T
Chemical processes influence the mechanical properties of geomaterials, resulting in either strengthening or weakening effects, the latter being particularly critical for long-term safety assessment in civil and energy engineering. Coupling of chemical and mechanical processes in cemented soils and rocks is investigated starting form a micro-structural chemo-mechanical model. The model consists of an assembly of grains and bonds undergoing dissolution or precipitation of mineral mass, affecting geometrical characteristics of the assembly. The principal such characteristics are the evolution of specific surface area and of bond cross-sectional area at the micro-scale, and of porosity at the macro-scale, which become key variables linking the micro-scale and macro-scale mechanisms. This framework has the advantage of avoiding unphysical situations, such as the occurrence of mineral precipitation with no pore space available or the occurrence of dissolution with no cementing material left. The evolution of important micromechanical quantities, such as the number of active bonds and their cross section is tracked. At the macro-scale, a reactive chemo-plasticity model is combined with a model for bonded geomaterials. The resulting micro- to macro-scale transition, schematically applicable to both materials with reactive grains and bonds and materials with only reacting bonds, is validated against the available experimental evidence, concerning calcarenite with both reactive bonds and grains made of the same mineral. The model is thus shown to provide a flexible framework for a consistent interpretation of experimental loading paths, and can be readily extended to more complex circumstances.
\end{abstract}

(c) 2015 Elsevier Ltd. All rights reserved.

\section{Introduction}

Terzaghi ${ }^{1}$ in his paper on Mechanism of Landslides discusses "landslide producing processes" where he distinguishes between "causes" and "contributing factors" of landslides. In particular, he states, "if a slope is old, heavy rainstorms (...) can hardly be the sole cause of a slope failure, because it is most unlikely that they are without any precedent in the history of the slope. They can only be considered contributing factors". Further ahead, in Table 1, among several "modes of action of agent" linked to water as the "acting agent" from heavy rain or snow melt, Terzaghi lists at \#9 the chemical weathering which "weakens intergranular bonds" leading to decrease in cohesion. For "old slopes", he then shows a progressive decrease in Factor of Safety, over 15 years, with some local ups and downs corresponding to dry and wet spells, reaching 1 , in conjunction with "a contributing factor" of high pore water pressure. He returns to this mechanism in the case of loess, where

\footnotetext{
* Corresponding author.

E-mail address: francesco.cecinato@unitn.it (F. Cecinato).
}

water from external reservoirs is listed as removing soluble binders destroying intergranular bond(s), with the same macroscopic effect of a decreasing cohesion.

Sensitivity of basic mechanical soil properties to chemical processes in the environment has been seen to become a critical factor of stability of slopes and coastal structures for a variety of reasons, from periodic changes in salinity of pore water affecting clay behavior of coastal slopes ${ }^{2,3}$ to oxidation and dissolution of sandstone, ${ }^{4}$ to dissolution of calcite, ${ }^{5}$ dissolution of silica in aging sediments, ${ }^{6}$ dissolution of gypsum in abandoned mines, ${ }^{7}$ to mention just a few examples.

In a more modern day context one needs to add that the dissolution of cementation bonds indicated by Terzaghi ${ }^{1}$ may be dramatically enhanced and accelerated by an increase in acidity of the run-off water, namely from an acid rain. In a case study of two landslide slip surfaces at Diao Jiao Zui and Qian Jiang Ping sites in the Three Gorges area, China, geochemical testing revealed that due to acid rain (with $\mathrm{pH}$ between 5.4 and 3.45) potassium ions at the slide surface were released, the cementation was reduced, and the ratio of interlayer clay minerals evolved. Accordingly, illite or 
Table 1

Parameter values adopted for model validation simulations.

\begin{tabular}{|c|c|c|c|c|}
\hline Parameter & Symbol & Set \#1 & Set \#2 & Units \\
\hline Initial porosity & $n_{0}$ & 0.56 & 0.56 & \\
\hline $\begin{array}{l}\text { Elastic modulus of uncemented solid } \\
\text { skeleton }\end{array}$ & $E_{g}$ & 1 & 1 & $\mathrm{MPa}$ \\
\hline Elastic modulus of cement material & $E_{b}$ & 150 & 150 & GPa \\
\hline $\begin{array}{l}\text { Poisson ratio of solid skeleton and } \\
\text { bonds }\end{array}$ & $\nu_{g}, \nu_{b}$ & 0.053 & 0.053 & \\
\hline Critical state parameter & $M_{C v}$ & 1.51 & 1.15 & \\
\hline Initial mean radius of grains & $R_{g}$ & 0.05 & 0.05 & $\mathrm{~mm}$ \\
\hline $\begin{array}{l}\text { Initial mean radius of bond cross- } \\
\text { sections }\end{array}$ & $R_{b}$ & 0.009 & 0.0105 & $\mathrm{~mm}$ \\
\hline Mean bond length & $d$ & 0.008 & 0.008 & $\mathrm{~mm}$ \\
\hline $\begin{array}{l}\text { Destructuration plastic strain } \\
\text { parameter }\end{array}$ & $A$ & 0.8 & 0.8 & \\
\hline $\begin{array}{l}\text { Mechanical destructuration rate } \\
\text { parameter }\end{array}$ & $k_{1}$ & 0.8 & 30 & \\
\hline Chemical healing rate parameter & $k_{2}$ & 0 & 0 & $\mathrm{~m}^{3} / \mathrm{kg}$ \\
\hline Slope of normal consolidation line & $\lambda$ & 0.05 & 0.025 & \\
\hline Number of grains & $N_{g}$ & $8.0 \times 10^{11}$ & $8.0 \times 10^{11}$ & $\mathrm{~m}^{-3}$ \\
\hline Number of bonds & $N_{b}$ & $=11 \times N_{g}$ & $=11 \times N_{g}$ & $\mathrm{~m}^{-3}$ \\
\hline Initial number of active bonds & $N_{b a}$ & $=N_{b}$ & $=N_{b}$ & $\mathrm{~m}^{-3}$ \\
\hline Tensile strength of cement material & $\sigma_{r t}$ & 8 & 8 & $\mathrm{MPa}$ \\
\hline $\begin{array}{l}\text { Compressive strength of cement } \\
\text { material }\end{array}$ & $\sigma_{r c}$ & 90 & 90 & $\mathrm{MPa}$ \\
\hline Uncemented preconsolidation stress & $p_{c}$ & 300 & 50 & $\mathrm{kPa}$ \\
\hline Constitutive parameter & $\beta$ & 2 & 2 & \\
\hline Constitutive parameter & $\gamma$ & 0.67 & 0.67 & \\
\hline Constitutive parameter & $\delta$ & 0.5 & 0.5 & \\
\hline Constitutive parameter & $\theta$ & 3.5 & 3.5 & \\
\hline Constitutive parameter & $a_{r 0}$ & 1 & 1 & $1 / \mathrm{mm}$ \\
\hline
\end{tabular}

montmorillonite-illite mixtures were transformed into montmorillonite associated with a strength change, including a $30 \%$ drop in shear strength. ${ }^{8}$

Further, the recent interest in geological $\mathrm{CO}_{2}$ sequestration in carbonate rocks brings challenges in predicting the effectiveness of the process, since the injected $\mathrm{CO}_{2}$ dissolves into an in-situ brine and produces also a weak acid, which in turn reacts with the surrounding carbonate rock (e.g. see $^{9,10}$ ). On the other hand, the technique of acid softening of rocks prior to hydraulic fracturing in unconventional gas and oil recovery or heat recovery from geothermal reservoirs poses interesting questions in ensuring the control of the process. ${ }^{11}$

The aim of this paper is to investigate a coupling of chemical and mechanical processes involved in weakening or strengthening of bonded geomaterials by means of a micro-structure inspired model. The micro-scale model is calibrated on (but it is not limited to) a regular array of grains and bonds undergoing dissolution or deposition of mineral mass. Only reactions of dissolution and precipitation between pore fluid and solid minerals are considered for the moment, neglecting complex phenomena involving solidsolid mass exchange (between bonds and grains, or between different sized minerals like in the Ostwald ripening phenomenon, $\mathrm{cf}^{12}$ ). Rate equations for mineral dissolution and precipitation are considered as known. ${ }^{13,14}$ Variable geometrical characteristics of the micro-structure are used to determine the evolving specific surface area $\left(\right.$ see $\left.^{6,15}\right)$ and the bond resisting cross-sectional area at the micro-scale, and variable porosity at the macro-scale, which become key variables linking the micro-scale and macro-scale mechanisms. At the macro-scale, a reactive chemo-plasticity model $^{17,18}$ is combined with a model for bonded geomaterials. ${ }^{19-21}$

Based on the kinetics of dissolution/deposition reaction of different minerals, two limiting cases can be considered, namely the case of both reactive grains and reactive bonds (e.g. calcarenite, where both grains and bonds are made of calcium carbonate) and the case of non-reactive grains and reactive bonds (e.g. silicic sand with carbonate bonds). While in the former case the timescales of dissolution/deposition of grains and bonds are comparable, in the latter case the timescale of reaction of the grains is orders of magnitude larger compared to that of bonds. In this work, due to the wide availability of relevant experimental evidence for validation, emphasis is put on the case of both reactive grains and bonds. However, the proposed framework could be easily extended to other cases, e.g. situations involving the presence of different families of cementation bonds characterized by different strengths and chemical dissolution properties.

The framework presented here aims at realistically reproducing the changes in the microscopic geometry of saturated bonded geomaterials due to both chemical dissolution/deposition and mechanical degradation, and consistently using them to quantify the macroscopic chemo-mechanical behavior of the material. This approach has the advantage of naturally providing the two limits of null dissolution (when cementing mineral is completely depleted) and null precipitation (when the porosity is null). The presented framework is not specialized for a particular geomaterial but aims at being more wide-ranging than existing works, and can be suited to different material types and modeling needs upon modifying key parameters.

Two types of data can be obtained using the existing technologies: data on the material strength change (decline) and on stiffness change, due to both the mechanical loading and chemical dissolution - that is typically provided by macro-scale experiments; and data about the chemical processes of dissolution and precipitation of the key minerals, which is obtained from microscale observation. Consequently, we are in the position to construct a two-scale model, which includes: (i) a macro-scale chemo-elasto-plastic model, where both the apparent preconsolidation stress and the apparent isotropic tensile strength depend on the material microstructural features, and (ii) an integrated model for mass change of minerals, in the framework of an evolving idealized micro-scale structure of grains and bonds, subject also to mechanical failure and chemical healing.

An example of a 2D material micro-scale structure is sketched in Fig. 1, inspired by micrographs of thin sections of calcarenite (e.g. see ${ }^{31}$ ), showing a gradual loss of mass and the consequent porosity increase due to chemical dissolution of both grains and bonds.

A constitutive model for the mechanical behavior of a reactive geomaterial that makes explicit use of a kinetic equation of a chemical reaction for a mineral, needs to be associated with a volume fraction of such mineral within a unit volume of soil. In the considered case it is assumed that bonds can be clearly identified at the micro-structure, and are made of a single mineral. The macro-scale is described by continuum variables of (elastic and plastic) strain and stress tensors, porosity, continuum free energy, and compressive and tensile strength. Such variables all depend on a series of macro-scale variables, such as the mass change of the minerals, and on micro-structural variables, as the geometry of bonds and grains, through phenomenological cross-scale functions, or averaging, or homogenization procedures.

In Section 2, the key literature evidence about chemo-mechanical coupling is discussed, both from the experimental and the constitutive modeling point of view. In Section 3 the macro-scale model is outlined, in terms of the key elasto-plastic features and of the macroscopic rate equations, defining the couplings between the mechanical and chemical behavior of the material. Section 4 is devoted to describing the derivation and calibration of cross-scale functions, enabling us to perform chemo-mechanical macroscale calculations accounting for the microstructure, while in Section 5 guidance is provided in the selection of constitutive parameters and initial values, with emphasis on the newly introduced variables compared to existing models. Finally, in Section 6 the model 

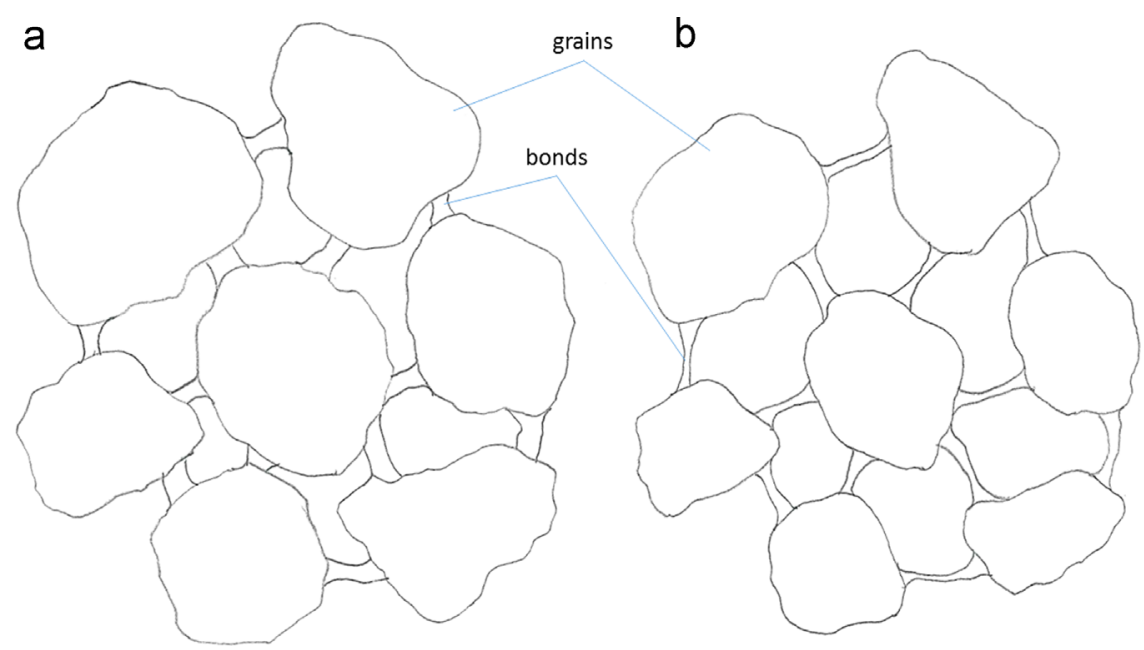

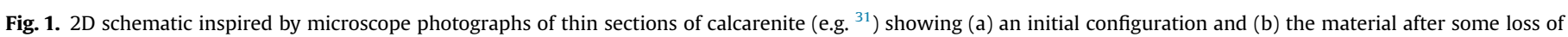
mass due to chemical dissolution of both grains and bonds, resulting in a porosity increase.

is validated by simulating a number of experimental chemo-mechanical loading paths reported in the literature, and conclusions are drawn in Section 7.

\section{Phenomenological and modeling background}

A common mechanism of chemical weakening or strengthening of soil is related to dissolution or precipitation of minerals, mainly calcium carbonate or silicates from and into intergranular bonds. In particular, dissolution of intergranular bonds as a result of water inundation, often in concomitance with dissolution or transformation of illite into smectite, or in the presence of highly acidic water, are considered as particularly adverse conditions. This is especially because little attention is paid to geochemical aspects of soil's mechanical evolution. ${ }^{1,22,23}$ Intergranular bonds often constitute a crucial and critical element of the so-called peak (undisturbed) material strength reached through shear or tensile loading, subject to axial or isotropic stress states, and possible drop of strength as a result of what is known as destructuration. In terms of micro-structural evolution, this may mean the rupture of certain populations of bonds, under a class of stress modes. Debonding is interpreted as a progressive failure of individual bonds, so that the overall process after its inception is relatively smooth. Debonding processes also affect elastic material properties. Because of bond breaking, the overall elasticity may be significantly reduced in shear, while the bulk (isotropic) modulus (in compaction) may be either increased or decreased. Viewed as a dependence of macroscopic elasticity on the amount of plastic deformation from the stand point of elasto-plasticity, this amounts to elasto-plastic coupling ${ }^{24,25}$ or if the process induces elastic anisotropy, to plasticity dependent elastic anisotropy. ${ }^{26-29}$

There is a relatively modest collection of data reported on the loss of strength at the macro-scale because of dissolution of key minerals. Castellanza and $\mathrm{Nova}^{30}$ report oedometer test results demonstrating changes in apparent preconsolidation pressure upon exposure to a weak acid solution $(\mathrm{pH}=2.8)$ both in calcarenite and in an artificial material made of silica sand bonded by lime. Remarkably, they measured a gradual, asymptotic decrease of apparent preconsolidation stress in calcarenite up to $70 \%$ of the original strength, increasing with the time of exposure to acid (cf. Fig. 12 of $^{30}$ ). For the same group of calcarenites, Ciantia et al. [31] found a drop in uniaxial compressive strength of the order of onefourth to one-third, upon wetting by respectively water and water with acid (with $\mathrm{pH}$ between 3.75 and 2.25). In the same experiments, a tensile strength reduction is also observed in water-inundated specimens of up to $26 \%$. Notably, also Young's modulus is observed to decline by up to $60 \%$.

Experimental data are also available about the loss of strength of bonded geomaterials due to destructuration, i.e. bond breakage due to mechanical loading. In this context, a significant set of results is reported in Lagioia and Nova, ${ }^{36}$ where the behavior of calcarenite under isotropic and triaxial loading paths is explored. All tests show the occurrence of a well-defined destructuration phase during loading, in which the material undergoes transition from rock-like to soil-like material at constant stress. Lagioia and Nova $^{36}$ also lay the foundations of a mathematical model for bonded materials that has subsequently seen further developments by the same group of authors.

From the constitutive modeling point of view, the mechanics of bonded geomaterials have been investigated through an extended version of the Cam Clay model (e.g. ${ }^{19,36,21}$ ), in which a plastic strain dependent isotropic tensile strength function is included, either coupled or decoupled from the classical preconsolidation hardening function. Bonded materials are often viewed as materials in a "natural state" with a structure, as opposed to materials in a "destructured" or "remolded" state. The latter state is reached via a process of destructuration, which is characterized by a gradual (as opposed to brittle) loss of strength, both in terms of isotropic tensile strength and shear strength as a result (and thus as a phenomenological function) of plastic straining. These mechanisms are conceived in the frame of Cam-clay plasticity and/or bounding surface plasticity. ${ }^{37-39}$ However, the above cited works are purely macroscopic, and apply to chemically inert environments. Coupled chemo-plasticity models have been developed either as a single scale or multi- scale models. Principles of singlescale models, formulated at the macroscale, are discussed in, $40,17,18,41$ with focus on the chemo-hydro-mechanical behavior of saturated clay materials. Hu and Hueckel ${ }^{11}$ proposed a three-scale model for granular materials linking irreversible damage strain with associated generation of micro-cracks, to mineral dissolution, from the individual grain level to the meso-scale (an aggregation of a few grains) to the macro-scale representing a large assembly of grains. Ciantia and Hueckel ${ }^{5}$ further built up on this three-scale concept, developing a model for the long-term weathering processes in calcarenite rocks, calibrated by reproducing compression tests in samples exposed to different degrees of acid weathering. 


\section{Macro-scale model}

\subsection{Yield function and hardening law}

The macroscopic constitutive model is developed assuming that the material behavior is isotropic, undergoing small strains, and the solid constituents are assumed incompressible. Only the features of the model that differ significantly from the standard critical state framework (e.g., ${ }^{42}$ ) are presented hereafter. The yield function is proposed along the lines of an existing approach for bonded geomaterials (e.g. ${ }^{19-21}$ ), in which an isotropic tensile strength function is included, as an extension of the modified Cam Clay model. ${ }^{43}$ The expression of the yield locus written in generalized stress space (compressive stress is here taken as positive) is

$$
\begin{aligned}
& F\left(\boldsymbol{\sigma}, \boldsymbol{\varepsilon}_{p}, a_{b}\right) \\
& =\left[M_{c v} L(\theta)\right]^{2}\left[\left(p+p_{\text {tens }}\right)^{2}-\left(p+p_{\text {tens }}\right)\left(p_{c}+p_{\text {comp }}+p_{\text {tens }}\right)\right] \\
& \quad+q^{2}=0
\end{aligned}
$$

where $M_{c v}$ is the critical state (i.e., constant volume) parameter, $L(\theta)$ is a function of the Lode angle describing the deviatoric section of the yield surface (in this work the function proposed by Willam and Warnke, ${ }^{44}$ has been used) , $p=I_{\sigma} / 3$ is the mean effective pressure, where $I_{\sigma}$ the first invariant of the effective stress tensor $\boldsymbol{\sigma}$ (note that the usual prime is omitted for brevity), $q=\sqrt{3 J_{2 \sigma}}$ the deviatoric stress, where $J_{2 \sigma}$ the second invariant of the deviatoric part of $\boldsymbol{\sigma}$ (e.g. see ${ }^{45}$ ), and $p_{c}$ is the (positive) preconsolidation pressure of the uncemented soil. The two positive macroscopic quantities $p_{\text {tens }}$ and $p_{\text {comp }}$ respectively represent the increase of tensile and compressive strength, compared to uncemented soil, due to the presence of cementation bonds. Coupling with the micromechanical behavior is introduced here, by assuming that

$p_{\text {tens }}=a_{b} \sigma_{r t}$ and $p_{\text {comp }}=a_{b} \sigma_{r c}$

where $\sigma_{r t}$ and $\sigma_{r c}$ are the macroscopic tensile and compressive strength (or the Euler stability stress, if Euler stability of bonds is taken into account) of the material constituting the cementing bonds, while $a_{b}$ is the mean specific cross section area of all mechanically active cementing bonds (corresponding to unbroken bonds). The macroscopic variable $a_{b}$ (with units $\mathrm{m}^{2} / \mathrm{m}^{2}$ ) depends on microscale quantities through an important cross-scale function discussed in detail in Section 4.1, and represents the total area of bonds in the porous skeleton's cross section per unit cross sectional area, only accounting for those bonds that are not broken, thus 'actively' contributing to the macroscopic strength.

It should be noted that when the material is completely destructured (i.e., with no active cementation bonds left), $a_{b}=0$, thus $p_{\text {tens }}=0$ and $p_{\text {comp }}=0$, hence the modified Cam Clay yield locus is recovered from Eq. (1). In Fig. 2 the evolution of the yield surface with cementation/destructuration is shown in terms of invariants $(p, q)$. It should be observed that the line of slope $M_{c v}$ (assumed independent of chemical effects for the sake of simplicity), passing by the yield locus' horizontal tangent point, actually identifies critical state only in the uncemented soil configuration, whereas in all other cases it represents a stress state at nil plastic volumetric strain rate, while the size of the yield locus can continue to evolve.

It is assumed that the yield surface crosses the isotropic stress axis in the zone of tension (negative value of mean pressure) at $-p_{\text {tens }}$ and in compression (positive values of mean pressure) at $\left(p_{c}+p_{\text {comp }}\right)$ (cf. Fig. 2), where the macroscopic preconsolidation pressure $p_{c}$ can be expressed as

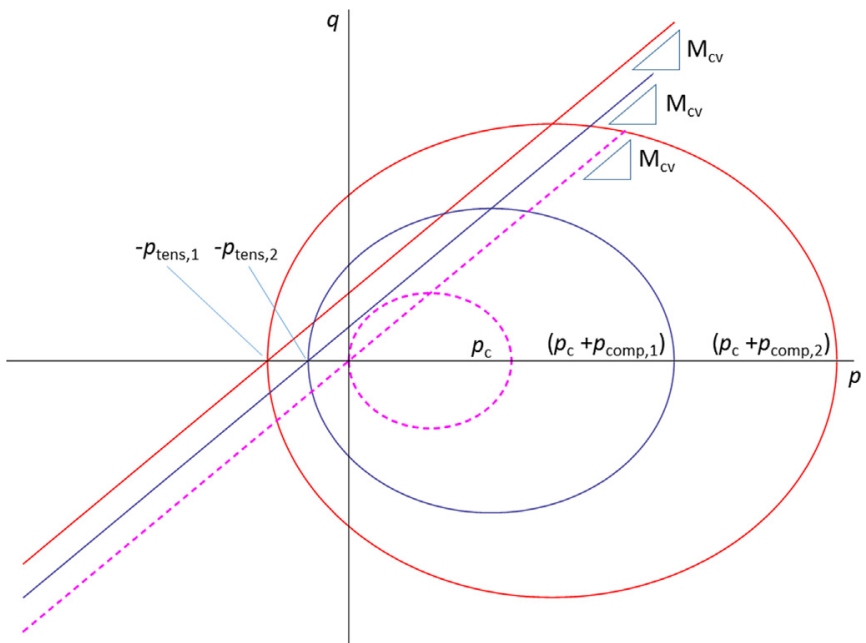

Fig. 2. Change of size of yield surface with changing cementation/destructuration (i.e. at different values of $p_{\text {tens }}$ and $p_{\text {comp }}$ ) in terms of invariants $(p, q)$. The dashed surface represents the uncemented state, in which $p_{\text {tens }}=p_{\text {comp }}=0$.

$p_{c}=p_{0} \exp \left\{\frac{1+e_{0}}{\lambda}\left[\varepsilon_{p}^{v}-\varepsilon_{0}\right]\right\}$

where $p_{0}, \varepsilon_{0}$ and $e_{0}$ are the reference mean pressure, volumetric strain and void ratio respectively, $\varepsilon_{p}^{v}$ is the plastic volumetric strain and $\lambda$ is a constitutive parameter. Eq. (3) provides a simplified hardening relationship similar to that of Cam Clay, which has been simplified by assuming negligible elastic compressibility. However, the proposed constitutive framework is not limited to such simple assumptions. The preconsolidation pressure defined in Eq. (3) is representative of the hardening state of the material in its unbonded condition, and directly depends on the accumulated volumetric plastic strain $\varepsilon_{p}^{v}$. In addition, the coupling with micromechanical behavior introduced with Eq. (2) implies the dependence of both macroscopic functions $p_{\text {tens }}$ and $p_{\text {comp }}$ on the number of mechanically active bonds, whose rate of change is bound to depend on both plastic straining (mechanical macro-scale effect) and on mass deposition/dissolution (chemical micro-scale effects), as shown in Section 4.1 (Eq. (7)).

\subsection{Elastic behavior}

The macroscopic strain tensor, $\boldsymbol{\varepsilon}$, is decomposed into additive elastic and plastic parts, $\boldsymbol{\varepsilon}_{e}, \boldsymbol{\varepsilon}_{p}$. The macroscopic elastic behavior of the material is described in the framework of hyperelasticity and elasto-plastic coupling, ${ }^{24,25}$ and can thus be deduced upon defining a suitable elastic free energy density function. To account for the presence of both mechanically and chemically interacting bonds and grains, the standard linear elasticity form of the free energy function of the uncemented solid skeleton (i.e. the unbonded soil) $\varphi_{g}=\varphi_{g}\left(\varepsilon_{e}\right)$ is modified, based on phenomenological interpretations, into a more general function:

$\varphi=\varphi\left(\varepsilon_{e}, \varepsilon_{p}, m_{b}, m_{g}\right)$.

Macroscopic variables $m_{b}$ and $m_{g}$ (expressed by the cross-scale functions introduced in Section 4.1) are the time-integrated mass change of all mechanically active cementing bonds and of grains, respectively, per unit volume, from a reference configuration $m_{b 0}, m_{g 0}\left(\Delta m_{b}, \Delta m_{g}>0\right.$ implies an increase of solid mass with respect to the reference configuration). For the sake of simplicity, since the model will be validated against chemo-mechanical degradation experiments (Section 6), a detailed description is provided here only for chemo-mechanical elastic weakening of the material, i.e. only the role of mechanical destructuration and 
chemical dissolution effects in the material elastic behavior are described. The more complex effects of chemical deposition, causing elastic strengthening, can be represented by a step-wise function that is not discussed in this work, for the sake of brevity.

We postulate that the macroscopic elastic free energy for a material with both reacting grains and bonds is expressed by

$\varphi\left(\varepsilon_{e}, \varepsilon_{p}, m_{b}, m_{g}\right)=(1-\tilde{\omega}) \varphi_{g}\left(\varepsilon_{e}-\frac{\left\langle-\Delta m_{g}\right\rangle}{\rho_{s}} \mathbf{I}\right)+\tilde{\omega} \varphi_{b}\left(\varepsilon_{e}\right)$

where $\varphi_{g}$ is the free energy of the uncemented solid skeleton, $\varphi_{b}$ is the free energy of the completely cemented soil (implying a larger stiffness than that of the unbonded material, since the whole porous space is filled with cement), $\mathbf{I}$ is the second order identity tensor, $\Delta m_{g}=m_{g}-m_{g 0}$ the current change of specific mass of grains, $m_{g}$, with respect to the initial value $m_{g 0}$. The weighting function $0 \leq \tilde{\omega} \leq 1$ is set to be a function of the value of the mean specific cross section area of mechanically active bonds $a_{b}$ (that depends on both cement mass change and plastic strain), as $\tilde{\omega}=a_{b}{ }^{\alpha}$, where $\alpha$ is a positive constitutive parameter. In this way, both elasto-plastic and chemical couplings are taken into account. Note that Macaulay brackets $\left\langle-\Delta m_{b}\right\rangle$ are used because macroscopic volume strains are induced by chemo-mechanical coupling only in the case of grain dissolution (and not of cement deposition).

Notably, Eq. (5) accounts for the occurrence of some macroscopic volumetric strain induced by grain dissolution. In fact, in the limit of null active cementing bonds (i.e. for small values of $\tilde{\omega}$ ), grain dissolution is associated to volume compression (due to the decrease of grain volume). The volumetric effect of grain dissolution in uncemented soil is completely analogous to the volumetric effect of a decrease of temperature in the grain material, in contrast with the effect of deposition, which is assumed to produce no volumetric strain. It should be however remarked that macroscopic volume strains induced by chemical dissolution are expected to be important only in the case of small volume of active bonds (i.e., when $\tilde{\omega}$ is small).

In addition to, and enhanced by the chemical degradation, the structure of the material may suffer from a mechanically induced destructuration. This is measured by plastic strain and brings about a reduction of the number of mechanically active bonds, resulting in the reduction of $a_{b}$, mean specific cross section area of mechanically active bonds, and hence of $\tilde{\omega}=a_{b}{ }^{\alpha}$. As a result, the first term of expression (5), representing the elastic energy of the destructured material (i.e. without any bonds), will gain larger relative importance, thus the overall material stiffness will decrease. When by subsequent chemo-mechanical evolution all the active cement bonds are broken $a_{b}=0$, then the free energy of the uncemented solid skeleton $\varphi_{g}$ is recovered. Whereas, when the porous material is completely cemented, $a_{b} \rightarrow 1$, the free energy becomes that of completely cemented soil $\varphi_{b}$.

From the chemical point of view, during cement dissolution a fraction of cement bonds, which in a general situation carries a fraction of the overall stress, disappears, causing the reduction of $a_{b}$. This leads to a stress increase in the remaining fractions of the bonds, thus inducing strains at constant stress level. As a result, cement dissolution induces a decrease of soil stiffness with associated strains (if dissolution occurs at constant stress) or a decrease of soil stiffness with an associated decrease of applied stress (if dissolution occurs at constant strain). These effects are adequately represented by Eq. (5).

It should be finally remarked that in Eq. (5) the compressibility of the solid constituents has been neglected. Further, only stiffness degradation due to bond breakage is considered in Eq. (5), while the increase of stiffness due to compaction is neglected for simplicity. The latter would imply a dependence of $\varphi_{g}$ on the porosity of solid skeleton, without including the volumes of the bonds.

\subsection{Macroscopic chemo-mechanical rate equations}

From the macroscopic point of view, the net mass change $\dot{m}$ within the REV due to dissolution and/or deposition is controlled by chemical kinetics. The rate of these reactions is proportional to the specific reactive surface area $a_{r}$ (with units $\mathrm{m}^{2} / \mathrm{m}^{3}$ ) per unit volume of the reacting materials, which corresponds to the area per unit volume of all interfaces of the porous skeleton that are exposed to chemical interaction, i.e. the areas of bonds and grains that are actually in contact with the pore fluid, and is defined by another important cross-scale function (Section 4.1).

Once the specific reactive surface area has been determined (see Section 4), the chemical reaction rate can be generally described by the following kinetic equation for the rate of mass change (i.e. specific mass change per unit time) of a specific mineral " $i$ " (see e.g. ${ }^{48}$ )

$\dot{m}^{i}=a_{r}^{i} k^{i}\left(\mu_{s}^{i}-\mu_{f}\right)$

with $k^{i}$ a rate constant (with units ( $\left.\left.\mathrm{kg} \mathrm{mol}\right) /\left(\mathrm{m}^{2} \mathrm{~s} \mathrm{~J}\right)\right), a_{r}^{i}$ the specific surface area of each mineral (with units $\mathrm{m}^{2} / \mathrm{m}^{3}$ ), and $\mu_{\mathrm{s}}^{i}$ and $\mu_{f}$ the chemical potentials (with units of $\mathrm{J} / \mathrm{mol}$ ) of the reacting solid mineral " $i$ " and of the fluid respectively. The rate constants $k^{i}$ are well established for most minerals (see e.g. ${ }^{49}$ ). For the sake of simplicity, a single reacting mineral will be considered both in the bonds and in the grains.

It can be observed that upon mass changes, also the specific reactive surface area in general changes, due to the evolving geometry of the solids. Thus, at constant chemical potential difference (e.g. if in an open system the material is subject to flushing with an acid solution), the mass rate of change evolves with the microscopic geometry of the system, which is represented by $a_{r}$. It should be also remarked that open systems only are considered in this work, for the sake of simplicity, implying that the dissolved mineral mass is transported away by the pore fluid (by advectiondiffusion) and thus no saturation of the pore fluid solution is considered. Modeling the transport of species within and outside the REV is out of the scope of the present work.

Let us denote with $N_{b}$ (with dimensions of $1 / L^{3}$ ) the number of potential (i.e. broken and unbroken) bonds per unit volume of the porous skeleton. It should be remarked that not all bonds within the REV are mechanically active, due for instance to (partial) bond rupture consequent to mechanical loading. In fact, only the unbroken bonds will contribute to the macroscopic strength and stiffness of the material. Hence, we define the macroscopic quantity $N_{b a}$ as the number of mechanically active bonds per unit volume. $N_{b a}$ ranges between 0 and $N_{b}$ (i.e. $0 \leq N_{b a} \leq N_{b}$ ) with $N_{b a}=0$ when all bonds are broken, and $N_{b a}=N_{b}$ when no bond is broken. The quantity $N_{b a}$ depends both on the amount of bond ruptures induced by mechanical loading (i.e., destructuration) and on the deposition of cement at broken bonds (with the consequent curing of broken bonds). The following empirical cross-scale function is proposed for the rate of $N_{b a}$, in which the two mechanisms of destructuration and cement deposition are clearly distinguishable:

$\dot{N}_{b a}=-k_{1} N_{b a} \sqrt{(1-A)\left(\dot{\varepsilon}_{p}^{v}\right)^{2}+A\left(\dot{\varepsilon}_{p}^{s}\right)^{2}}+k_{2}\left(N_{b}-N_{b a}\right)\left\langle\dot{m}_{b}\right\rangle$

In the above, $A, k_{1}$ (dimensionless) and $k_{2}$ (with units $\mathrm{m}^{3} / \mathrm{kg}$ ) are constitutive parameters, with $A$ ranging between 0 and 1 and weighting the role of volumetric $\left(\dot{\varepsilon}_{p}^{v}\right)$ and deviatoric $\left(\dot{\varepsilon}_{p}^{s}\right)$ plastic strain rates in destructuration (i.e., decementation). The deviatoric plastic strain rate is defined as $\dot{\varepsilon}_{p}^{s}=\sqrt{(2 / 3) J_{2 \varepsilon}}$, where $J_{2 \varepsilon}$ the second invariant of the increment of the plastic deviatoric strain tensor 
(e.g. see ${ }^{45}$ ). The Macaulay brackets in the last term of Eq. (7) are needed because only cement deposition is assumed to form new active bonds, whereas plastic strains are assumed to induce only bond rupture. Positive $\dot{m}_{b}$ is associated with mass increase (due to deposition) of bonds.

As a result, in general cement dissolution/deposition $\dot{m}_{b}$ affects soil's mechanical strength in two ways, namely (i) through the restoration of previously broken bonds (with an increase of the number of active bonds $N_{b a}$, second term of Eq. (7)) and (ii) through the reduction/increase of the bond cross sections. In fact, in general cement dissolution/deposition $\dot{m}=\dot{m}_{g}+\dot{m}_{b}$ is expected to affect both porosity and the thickness of bonds. Thus, it is reasonable to assume that the fractions of $\dot{m}=\dot{m}_{g}+\dot{m}_{b}$ involving grains and bonds are proportional to the respective reactive specific surface areas $a_{r g}$ and $a_{r b}$, namely

$\dot{m}_{b}=\frac{a_{r b}}{a_{r b}+a_{r g}} \dot{m}$ and $\dot{m}_{g}=\frac{a_{r g}}{a_{r b}+a_{r g}} \dot{m}$.

Starting from the standard definition of porosity, upon chemomechanical loading, the rate of porosity change of the material is thus deemed to depend on both mass change and on volumetric strain, as follows:

$\dot{n}=-\frac{\dot{m}}{\rho_{s}}-(1-n) \operatorname{Tr}(\dot{\varepsilon})$

The above represents the equation of continuity for the porous skeleton (due to the assumption of incompressible solid constituents). Within the proposed chemo-mechanical framework based on small strains concepts, it is convenient to introduce here a 'small strain' porosity $\tilde{n}$ depending only on mass dissolution/ deposition and neglecting volumetric strain, through the following rate equation:

$\dot{\tilde{n}}=-\frac{\dot{m}}{\rho_{s}}$.

It should be remarked that $\dot{\tilde{n}}$ is simply a fraction of $\dot{n}$, thus $\tilde{n}$ and $n$ are not referred to different spatial configurations.

\section{Microscale description and micro-to-macro relationships}

In this section the cross-scale functions defining key macroscopic variables $a_{b}$ and $a_{r}$, enabling us to perform chemo-mechanical macroscopic calculations accounting for the microstructure, are derived in terms of microscopic variables, with reference to a simplified microscopic geometry. Cross-scale functions are then calibrated based on a rigorous microscopic geometry calculation assuming a cubical representative elementary volume (REV) as an example. However, these interpolation functions can be easily adapted to different micromechanical models.

In Fig. 3, a 2D idealization of the considered geometry at the microscopic scale (where the key dimensions of grains and bonds are indicated) inspired from the realistic schemes of Fig. 1 is shown, in the general case where grains might not be in direct contact, but they are linked by cementation bonds that are assumed to be isotropically distributed. In a large porosity configuration such as that illustrated in Fig. 3, the geometry of bonds can be approximated with that of a cylinder, and the geometry of grains can be represented by that of a sphere. This geometry is assumed to represent the reference configuration for materials where both grains and bonds are subject to the same rate of dissolution/deposition. In fact, it can be expected that at a generic instant of the dissolution/deposition history of such materials the grains appear linked by cement bonds but are not necessarily in direct contact (Figs. 1 and 3).

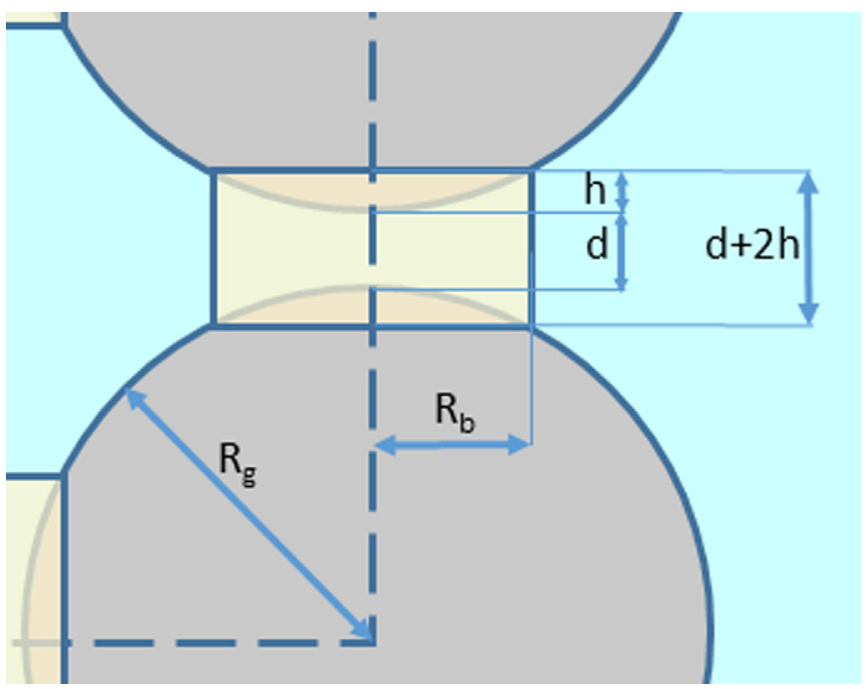

Fig. 3. Schematic of the considered simplified geometry at large porosity, in a general case where the spherical grains are not in direct contact, but they are linked by cylindrical bonds.

\subsection{Derivation of cross-scale functions}

Let us denote with $N_{g}$ (with dimensions of $1 / L^{3}$ ) the number of grains per unit initial bulk volume of the porous skeleton. With reference to the general case of Fig. 3, let $R_{g}$ be the mean radius of the grains, $R_{b}$ the mean radius of the bonds, and $L_{b}$ the mean length of the bonds, which is generally defined as $L_{b}=d+2 h$, where $d \neq 0$ the distance between the edges of two grains connected by a bond, and $h$ the height of the spherical sector wrapped by the bond, which is expressed as

$h=R_{g}-\sqrt{R_{g}^{2}-R_{b}^{2}}$.

According to the above introduced notation, we can easily deduce the specific volume of the grains $v_{g}=N_{g} \frac{4}{3} \pi R_{g}^{3}$ (expressed as $L^{3} / L^{3}$ and referred to the unit initial bulk volume) and that of the bonds (volume of the cylinders less the volume of spherical sectors wrapped by the bonds) as

$v_{b}=N_{b} \pi\left[R_{b} L_{b}^{2}-h\left(R_{b}^{2}+h^{2} / 3\right)\right]$.

Thus, the volume of the solid phase (per unit initial volume of the porous skeleton) coincides with

$1-\tilde{n}=v_{b}+v_{g}$

where $\tilde{n}$ the 'small strain' porosity (Eq. (10)), whereas the solid masses of the grains and of the bonds can be easily deduced by multiplying the specific volumes by the density of solids $\rho_{s}$.

The specific reactive surface area $a_{r}$ (per unit bulk volume, expressed in $L^{2} / L^{3}$ ) can be expressed as the sum of the specific area of grains and of bonds. The latter coincides with the sum of the lateral surface area of all the bonds and of the cross section areas of the broken bonds (which are $N_{b}-N_{b a}$ ), and is equal to

$a_{r b}=N_{b} 2 \pi R_{b} L_{b}+\left(N_{b}-N_{b a}\right) \pi R_{b}^{2}$

whereas the reactive specific surface area of the grains is given by

$a_{r g}=N_{g} 4 \pi R_{g}^{2}-N_{b} 2 \pi R_{b} h$.

Thus, the specific reactive surface area (of grains and bonds) is $a_{r}=a_{r g}+a_{r b}=N_{g} 4 \pi R_{g}^{2}-N_{b} 2 \pi R_{b} h+N_{b} \pi R_{b} L_{b}+\left(N_{b}-N_{b a}\right) \pi R_{b}^{2}$.

It should be observed that the expressions of specific reactive 
surface area given above are valid as long as the porosity is large, as exemplified in Figs. 1 and 3, because, when the volume of bonds and of grains is large (and the porosity becomes negligible), the reactive surface area is expected to become negligible. Indeed, at the end-phase of precipitation, when the volumes of growing neighboring bonds and grains start to overlap, details of the geometry of bonds become very intricate.

Hence, to relate the microstructure to the macroscopic behavior, instead of strictly following a given microscopic geometry, we introduce phenomenological restrictions to avoid physical inconsistencies in the system evolution. In particular, when the volume of precipitates fills all the available voids space, $n \rightarrow 0$ is enforced; and when all bonds are dissolved, i.e. $n \rightarrow 1$, the specific reactive surface area $a_{r}$ is imposed to tend to zero. When porosity tends to zero, a general asymptotic relationship between $a_{r}$ and $n$ holds:

$a_{r}=a_{r 0} n^{\gamma}$

where $a_{r 0}$ and the exponent $\gamma$ are constitutive parameters. The above can be obtained by assuming that, at the limit of very small porosity, the porous space is described by a given number of voids of given shape (e.g., spherical) per unit volume. Derivation of Eq. (17) is not illustrated in detail for the sake of brevity.

Combining Eqs. (16) and (17) with an appropriate weighting function $\omega_{1}$, we obtain the following relationship expressing the specific reactive surface area of materials with both reacting grains and bonds throughout the porosity range:

$$
\begin{aligned}
a_{r}= & {\left[N_{g} 4 \pi R_{g}^{2}-N_{b} 2 \pi R_{b} h+N_{b} 2 \pi R_{b} L_{b}+\left(N_{b}-N_{b a}\right) \pi R_{b}^{2}\right] } \\
& \left(1-\omega_{1}^{\beta}\right)+a_{r 0} n^{\gamma} \omega_{1}^{\beta}
\end{aligned}
$$

where $\beta$ is a constitutive parameter. We propose to express the weighting function $\omega_{1}$ as

$\omega_{1}=1-n$

The weighting function (19) $\omega_{1} \rightarrow 0$ for $n \rightarrow 1$, and $\omega_{1} \rightarrow 1$ for $n \rightarrow 0$, thus it effectively inhibits the exchange of cement mass (due to dissolution/precipitation) when there is no material left, or when there is no void volume left. It is worth highlighting that, for the sake of simplicity, Eq. (18) implicitly neglects the reactive surface area due to micro-cracks that are induced at constant porosity. As a result, at vanishing porosity $(n \rightarrow 0) a_{r} \rightarrow 0$, independently of the possible existence of micro-cracks.

It should be noted that the specific reactive surface is also affected by the number of bond ruptures induced by mechanical loading. In fact, mechanical loading can induce a decrease of the number of active bonds per unit volume $N_{b a}$ (as is discussed in Section 3.3), thus leading to an increase of reactive surface area $a_{r}$.

Moreover, the cementing bonds contribute to compressive, tensile and shear strength of the porous medium. Knowing the number of active bonds per unit volume $N_{b a}$, the number of active bonds per unit linear length can be expected to be of the order of $N_{b a}{ }^{1 / 3}$, hence the number of active bonds per unit initial cross section area of the porous skeleton (expressed in $1 / L^{2}$ ) can be approximately estimated as $N_{b a}^{2 / 3}$. The specific cross section area of cementing bonds $a_{b}$ (expressed as $L^{2} / L^{2}$ and referred to the unit initial cross section) can then be evaluated, at the limit of large porosity $(\tilde{n} \rightarrow 1)$, as

$a_{b}=\pi R_{b}^{2} N_{b a}^{2 / 3}$

and on the other hand, at the limit of very small porosity $(\tilde{n} \rightarrow 0)$, as

$a_{b}=1-\tilde{n}$

Applying for the specific cross section area of cementing bonds similar interpolation concepts to those adopted for the reactive specific area, we obtain

$a_{b}=\pi N_{b a}{ }^{2 / 3} R_{b}^{2}\left(1-\omega_{2}\right)^{\theta}\left(1-\omega_{3}\right)^{\delta}+(1-\tilde{n}) \omega_{2}{ }^{\theta} \omega_{3}{ }^{\delta}$

In the above, $\delta$ and $\theta$ are constitutive parameters, and the weighting functions $\omega_{2}$ and $\omega_{3}$ are proposed as

$\omega_{2}=1-\tilde{n}, \quad \omega_{3}=\left(\frac{v_{b}}{1-v_{g}}\right)$

where $\left(1-v_{g}\right)$ represents the specific volume (referred to the unit initial bulk volume) that is available for a complete cementation of bonds. Eq. (23) represents a function which has null value when $v_{b}=0$ (no cementing bonds), and equals one when growing precipitated bonds fill all the void space, i.e. for $v_{b}=1-v_{g}$ (no void volume). As a result, the specific cross section area of cementing bonds $a_{b}$ ranges between 0 (at large porosities) and 1 (at negligible porosity).

It is worth recalling that the use of $\tilde{n}$ (instead of $n$ ) comes from small strains theory and implies that under a purely elastic deformation (with no rupture of cementation bonds and no dissolution/deposition) $a_{b}$ (defined in Eq. (22) is independent of volumetric strains (as expected from a physical point of view). As a result, $a_{b}$ depends only on plastic strains (which can induce rupture of cementing bonds) and on cement deposition/dissolution.

\subsection{Calibration of cross-scale functions: example representation of micro-macro relationships}

Since the key equations of the model outlined in Section 4.1 are not tied to a specific geometrical configuration, the question arises as to how the constitutive parameters can be calibrated to represent a realistic (albeit simplified) situation. To validate our constitutive assumptions, an attempt is made here to describe the microscopic geometry in a rigorous manner throughout the porosity range. In other words, the evolution of the geometry at the microscale due to dissolution/deposition is tracked without resorting to the phenomenological simplifications described in Section 4.1. Instead, reference is made to a cubical REV composed of eight spherical grains (in contact or not, with one to another), where each grain is connected to six other grains by means of cylindrical bonds. The spherical grains are centered in the REV's vertices and the cylindrical bond axes lie in the REV's edges, so that the porous space is located at the center of the REV. The resulting trends obtained with the microscopic representation are then compared to the macroscopic model representation of Section 4.1, in order to evaluate the constitutive parameters $\beta$ and $\delta$ of the cross-scale functions Eqs. (18) and (22).

The reference configuration consists, as an example, of identical spherical particles that are linked by identical cylindrical bonds so that the center-to center of grains distance $H_{c}=d+2 R_{g}$. No bond breakage by mechanical action is considered in this example, but the geometry of the solid skeleton is changed as a result of uniform deposition/dissolution, so that the gain/loss of thickness is constant per unit area, throughout the solid skeleton's reacting surface.

The microscopic representation is subdivided into two subcases, corresponding to different geometrical configurations occurring during chemical interaction of the material: a first configuration, at larger porosity, in which both bonds and grains' surfaces are exposed to chemical interactions and thus undergo uniform thickness reduction/increase. This configuration is lowerbounded by the complete dissolution of bonds (when grains may spatially rearrange as they would lose support, but could keep undergoing further dissolution until $n \rightarrow 1$ ), and upper-bounded by the point where the growing grains (spheres) outpace the growing bonds (cylinders), i.e. the point at which growing cylinders are 


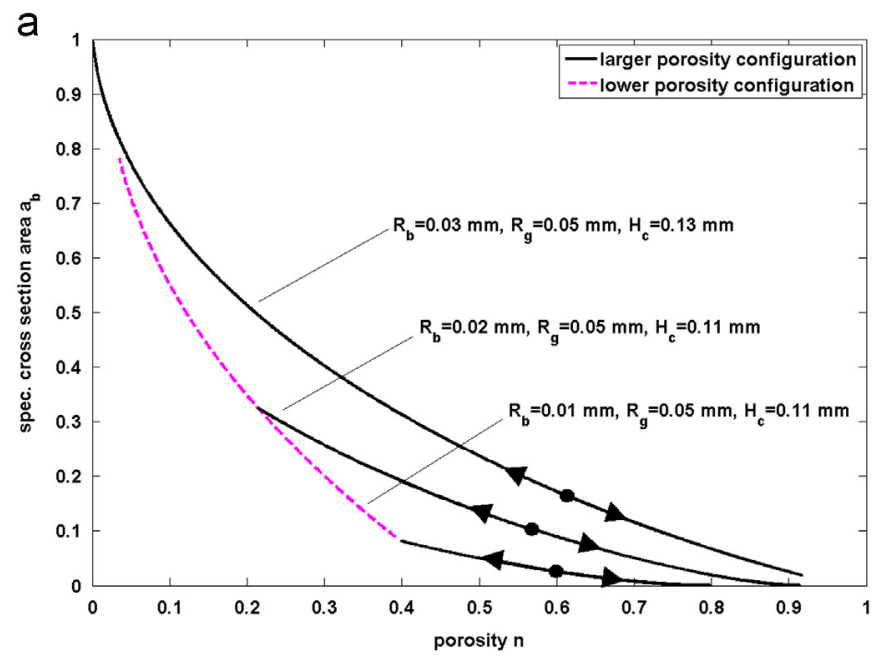

b

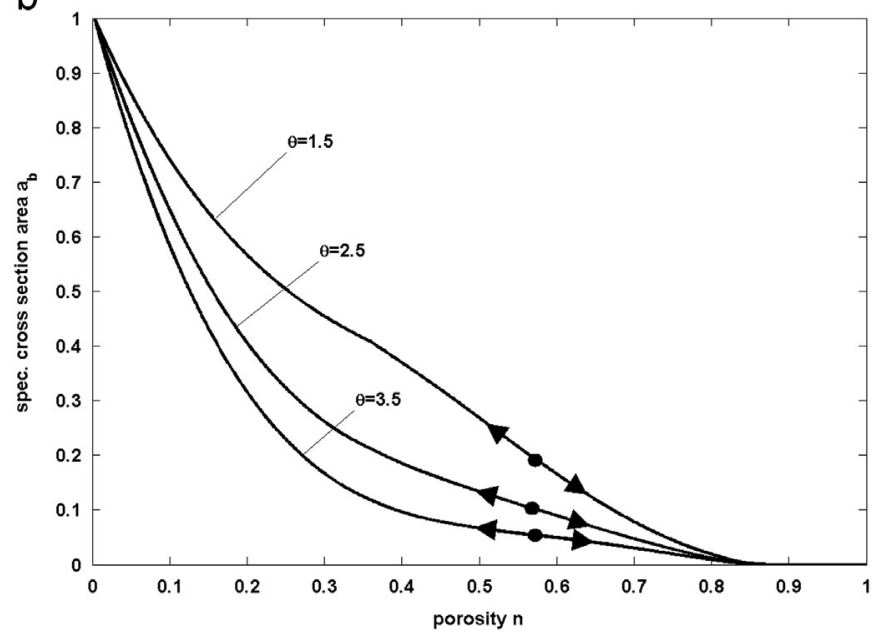

Fig. 4. Specific cross section area of cementing bonds versus porosity during dissolution/deposition (the dots mark the initial porosity): (a) microscale calculation according to three different intial geometrical configurations and (b) macroscopic calculation with model equation (22) for three different values of parameter $\theta$ and for $\delta=0.3$.

embedded into the growing spheres, due to faster overall rate of growth of the spherical shape compared to the cylindrical one. This happens when $R_{g}=\sqrt{{H_{c}}^{2} / 4+R_{b}^{2}}$. Further deposition beyond this point implies a second configuration, at lower porosity, where only grains grow, hence the skeleton geometry is represented by large, interpenetrating spheres. This second case is limited by the possible partial overlapping of spheres located at opposite positions along the REV diagonal. Beyond this point, further deposition would imply a possible residual very small porosity and a different geometry, which is not investigated here. Hence, the second case applies to the range $\sqrt{{H_{c}}^{2} / 4+R_{b}^{2}} \leq R_{g}<H_{c} \sqrt{2} / 2$.

Starting from an initial sample configuration where $R_{b}=0.02 \mathrm{~mm}, R_{g}=0.05 \mathrm{~mm}$ and $H_{c}=0.11 \mathrm{~mm}$, in Fig. 4a the specific cross section area of cementing bonds $a_{b}$ is plotted versus porosity, distinguishing by different line types the two sub-cases discussed above (in the second configuration, the cross section area at the intersection of overlapping spheres is used to compute $a_{b}$ ). The slope discontinuity in the curves represents the passage between the first and second geometrical configuration. Expectedly, it can be observed that $a_{b} \rightarrow 1$ when $n \rightarrow 0$ and $a_{b} \rightarrow 0$ when $n \rightarrow 1$. Sensitivity to the relative proportions of grains and bonds is also explored in Fig. 4a by plotting two extra curves characterized by different combinations of $R_{b}, R_{g}$ and $H_{c}$. In Fig. 4b

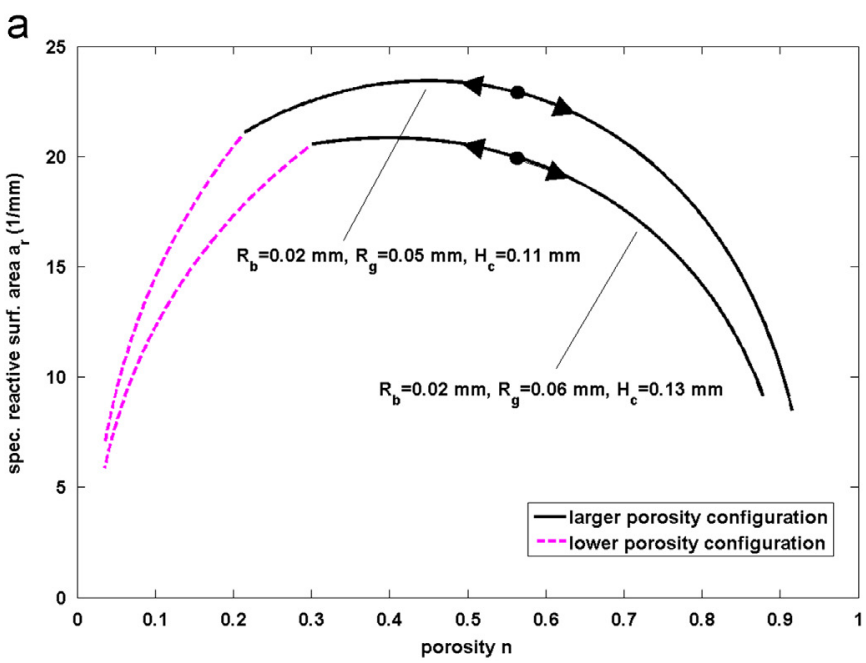

b

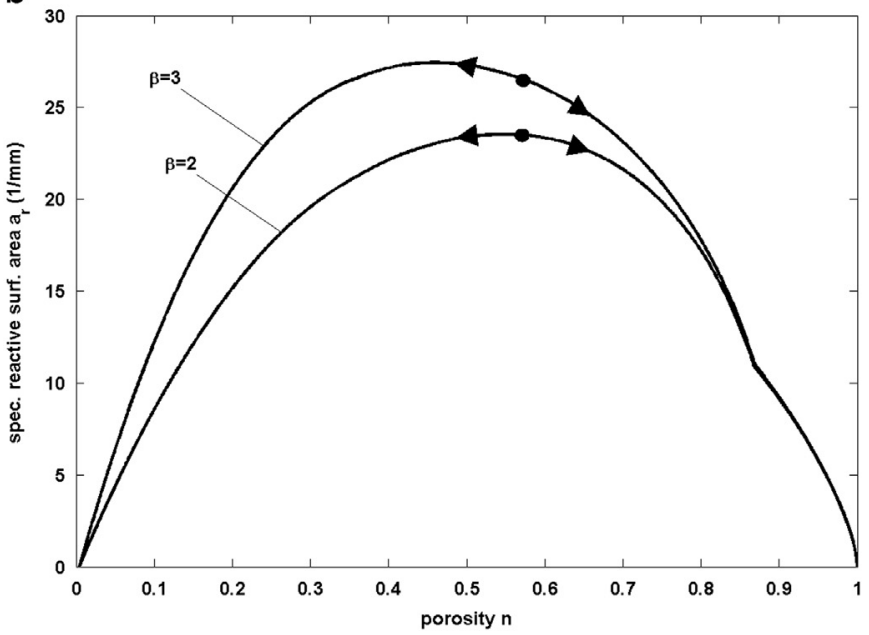

Fig. 5. Specific reactive surface area versus porosity during dissolution/deposition (the dots mark the initial porosity): (a) microscopic calculation according to two different intial geometrical configurations and (b) macroscopic calculation with model equation (18) for two different values of parameter $\beta$.

the equivalent curves obtained with the modeling approach described in Section 4 are shown, starting from the same geometrical configuration, where $N_{b}=6 N_{g}$, for different values of exponent $\theta$ (cf. Eq. (22)) and for $\delta=0.3$.

In Fig. 5a the specific reactive surface area $a_{r}$ obtained by microscopic calculation is plotted versus porosity, for two different combinations of initial $R_{b}, R_{g}$ and $H_{c}$ values. It can be noticed that, as expected, the specific reactive surface tends to zero at both limits of null and unit porosity. Correspondingly, in Fig. 5b the macroscopic calculation results obtained using model equation (18) are drawn for two different values of parameter $\beta$.

Comparison between the two sets of curves described above suggests that calculations of $a_{b}$ and $a_{r}$ respectively obtained with Eqs. (22) and (18) provide a satisfactory representation of the material's microstructure.

\section{Selection of constitutive parameters and initial values}

Compared to the standard critical state framework, additional parameters have been introduced in the micro-mechanically based constitutive model described above. Overall, the parameters featuring in this model can be grouped into four families, namely: four constitutive parameters describing the material's uncemented 
behavior $\left(\lambda, \phi_{c v}, E_{g}, \nu_{g}\right)$, three constitutive parameters describing the mechanical properties of the cementing material $\left(\sigma_{r c}\right.$ and $\left.\sigma_{r t}\right)$ and of completely cemented soil $\left(E_{b}\right)$, two constitutive parameters describing the mechanically-induced destructuration $\left(A, k_{1}\right)$ and one parameter to describe chemical healing $\left(k_{2}\right)$, and finally five parameters for describing the macroscopic evolution of specific reactive surface area and the specific cross section area of cementing bonds $\left(\beta, \gamma, \delta, \theta, a_{r 0}\right)$. The latter five parameters, resulting from the above presented, bespoke micro-to-macro formulation, constitute the most innovative aspect of the proposed model, compared to previously published works.

Furthermore, the model needs the definition of the initial values of a number of quantities. In particular, in addition to the usual definition of the initial porosity $n_{0}$ and of the uncemented preconsolidation pressure $p_{c}$, six new quantities are needed to describe the initial microscopic geometry, namely: the number of grains, of cementing bonds and of active bonds $\left(N_{g}, N_{b}, N_{b a}\right)$ and the geometric characteristics of the grains $\left(R_{g}\right)$ and of the bonds $\left(R_{b}, d\right)$.

In light of the above described features, it is useful to discuss how the constitutive parameters on the one hand, and the initial values of the quantities describing the initial conditions on the other hand, can be selected.

The number of grains $N_{g}$ can be selected from the solid mass of the grains (coinciding with the total solid mass minus the solid mass of the cementing bonds), the specific gravity of the grains and the mean grain radius $R_{g}$. The number of cementing bonds $N_{b}$ can be evaluated from $N_{g}$ and an estimate of the coordination number, which is assumed to correspond to the number of bonds per grain. The number of active bonds $N_{b a}$ ranges between 0 and $N_{b}$, and can be reasonably estimated to be equal to $N_{b}$ for an undamaged cemented material. All quantities mentioned above, together with the geometric characteristics of the grains and of the bonds, can be estimated from examining thin sections of the microstructure of the cemented material. Useful hints for the selection of the coordination number can be obtained from theoretical and experimental evaluations of the coordination number of random arrays of spheres in contact with each other (e.g. ${ }^{50}$ ). Such studies provide estimations of the coordination number of the order of $4-8$ at large porosities and 8-12 at low porosities. In any case, precious information can be also obtained from quantitative chemical analyses, providing for instance the variation of solid mass of the sample (coinciding with the mass of dissolved bonds and solid grains, if these are chemically active) at different degrees of chemical interaction, possibly related to representative thin sections of the evolving microstructure.

The elastic stiffness of the completely cemented soil $E_{b}$ can be reasonably approximated with that of the cementing material. Thus, the three constitutive parameters needed to describe the mechanical properties of cementing material $\left(E_{b}, \sigma_{r c}\right.$ and $\left.\sigma_{r t}\right)$ can be selected from the literature, making reference to the intact material constituting the cementing bonds. It is worth adding that from standard tests (unconfined compression tests, isotropic compression tests and Brazilian tests), using trial-and-error procedures, it is possible to estimate the two positive quantities $p_{\text {tens }}$ and $p_{\text {comp }}$ representing the increase of tensile and compressive strength, due to the presence of cementation bonds, and the value of the uncemented preconsolidation pressure $p_{c}$ (which should however be evaluated, if possible, also taking into account the behavior of the uncemented material). As a result, in order to avoid an over-constraint in the selection of $p_{\text {tens }}$ and $p_{\text {comp }}$ (due to the fact that $p_{\text {tens }}$ and $p_{\text {comp }}$ are evaluated from both micro-geometry quantities and macro mechanical experiments), the coordination number and the mechanical properties of the cementing material ( $\sigma_{r c}$ and $\sigma_{r t}$ ) need to be adjusted to achieve consistency between the two evaluations of $p_{\text {tens }}$ and $p_{\text {comp }}$.
Anyway, the evolution of $p_{\text {tens }}$ and $p_{\text {comp }}$ (from unconfined compression tests, isotropic compression tests and Brazilian tests) associated with the corresponding variation of the solid mass of the sample, at different degrees of chemical interaction, is certainly a valuable information for the precise calibration of the whole model.

The constitutive parameters describing the uncemented behavior $\left(\lambda, \varphi_{c v}, E_{g}, \nu_{g}\right)$ and the related initial value of the uncemented preconsolidation pressure $p_{c}$, should in principle be evaluated on the uncemented material (e.g. ${ }^{37}$ ). However, this is not always an easy task, thus these parameters are often evaluated by a trialand-error procedure performed on the cemented material only. This obviously involves a higher level of uncertainty in the evaluation of $p_{\text {tens }}, p_{\text {comp }}$ and of $p_{c}$.

Finally, the constitutive parameters describing mechanicallyinduced destructuration $A$ and $k_{1}$ can be evaluated from the postpeak response in triaxial compression and post-yield behavior in isotropic compression tests. In this case, however, great care must be taken to account for the possible occurrence of shear or compaction bands, that can deeply affect the conventional response of the sample, ${ }^{52,53}$ implying the impossibility to calibrate the model assuming a homogeneously deforming sample. Parameter $k_{2}$ describing chemical healing can be in principle calibrated on mechanical (e.g. uniaxial compression) tests carried out on samples of bonded geomaterial subjected to increasing degrees of cementation, although this parameter is not used in the model validation presented in Section 6 (see Table 1), since reference is made to chemo-mechanical weakening experiments only.

\section{Model validation}

The above described model was numerically integrated through a fully implicit, backward Euler integration scheme, showing adequate convergence. From the macroscopic, constitutive point of view, as specified in Section 3.1, an extended version of Cam Clay yield function and hardening relationship were implemented. An associated flow rule was adopted for the sake of simplicity.

The model capabilities to reproduce the experimental chemomechanical behavior of bonded geomaterials were tested by simulating a series of loading paths along the lines of experimental datapoints reported in the literature, after having deduced the relevant parameters as much as possible from published data.

\subsection{Simulation of chemo-mechanical loading paths}

To support validation of the model in simulating chemo-mechanical loading paths in materials with both reactive grains and bonds, reference can be made to the extensive set of experiments reported by Ciantia et al., ${ }^{31}$ Ciantia and Hueckel ${ }^{5}$ and Castellanza and Nova $^{30}$ on a number of samples of calcarenite subject to different combinations of acidic weathering and mechanical loading.

As a first example, the model is tested by simulating unconfined compression tests on saturated calcarenite, by first reproducing an acid weathering phase and subsequently a mechanical loading phase, and comparing the simulations with the experimental data of Ciantia et al. ${ }^{31}$ In this context, a variable expressing the 'degree of dissolution' can be introduced as $\xi=M_{\text {dis }} / M_{0}$, where $M_{\text {dis }}$ represents the dissolved mass and $M_{0}$ the initial mass of reacting solids, so that $\xi=0$ for the unweathered material and $\xi=1$ at complete dissolution of the reacting solids.

The parameter values adopted for this set of simulations on calcarenite are shown in Table 1 (set \#1). In particular, the initial average grain radius and bond radius and length were selected 
upon evaluating thin section photographs reported in $^{31}$. The initial porosity was chosen from a reference range of $0.4-0.6$, established from Table 1 of ${ }^{31}$.

As discussed in Section 3.2, the equivalent elastic modulus of the bonded material $E$ depends on both the elastic properties of calcite cement and of the unbonded granular material, and is calculated upon taking the second derivative of the free elastic energy density (5) with respect to elastic strain. Thus, we obtain an expression of the form

$E=E_{g}\left(1-a_{b}{ }^{\alpha}\right)+E_{b} a_{b}{ }^{\alpha}$

where $E_{g}$ and $E_{b}$ are the elastic moduli of uncemented solid skeleton and of completely cemented soil (which is approximated with that of pure calcite) respectively, and parameter $\alpha=1.8$. It should be noted that Eq. (24) is also consistent with mixture theory-based rules to obtain the equivalent parameters of a composite mass, typically employed in soil improvement calculations (e.g. see ${ }^{54}$ ). As initial value, estimating $E_{b} \approx 150 \mathrm{GPa}$ from sonic wave velocity data on calcite after ${ }^{55}$ and $E_{g} \approx 5 \mathrm{MPa}$, using (24) we obtain an equivalent initial elastic modulus $E_{0} \simeq 117 \mathrm{MPa}$.

To obtain the initial values of $p_{\text {tens }}$ and $p_{\text {comp }}$ via Eq. (2), and thus define the initial yield locus size, the values of unconfined compressive and tensile strength of calcite can be deduced from the literature. Goodman ${ }^{55}$ reports $q_{c}=90 \mathrm{MPa}$ and $q_{t}=q_{c} / 30$ respectively, for carbonate rocks. However, for a more realistic reproduction of experimental trends, also considering the data set on the compressive and tensile strength of a number of saturated calcarenite samples reported by Ciantia et al. ${ }^{31}$ (Table 1 ), we choose here $\sigma_{r c}=90 \mathrm{MPa}$ and $\sigma_{r t}=8 \mathrm{MPa}$.

\subsubsection{Chemo-mechanical uniaxial compression tests}

In Fig. 6, simulations of stress-strain curves during unconfined compression tests are plotted at different values of $\xi$, together with analogous experimental data. To adequately reproduce experimental conditions, the simulation consisted of two steps, namely of a first step reproducing exposure to an acid solution up to the target value of $\xi$, and of a second step simulating the unconfined compression test in the weathered material. The reproduction of experimental data is especially accurate until the ultimate strength is attained. After the peak, simulations exhibit a smaller amount of softening than experiments, overall showing a less markedly fragile behavior. Since the post-peak response is typically deeply affected by shear or compaction banding which leads the conventional behavior to be much different from that of a homogeneously

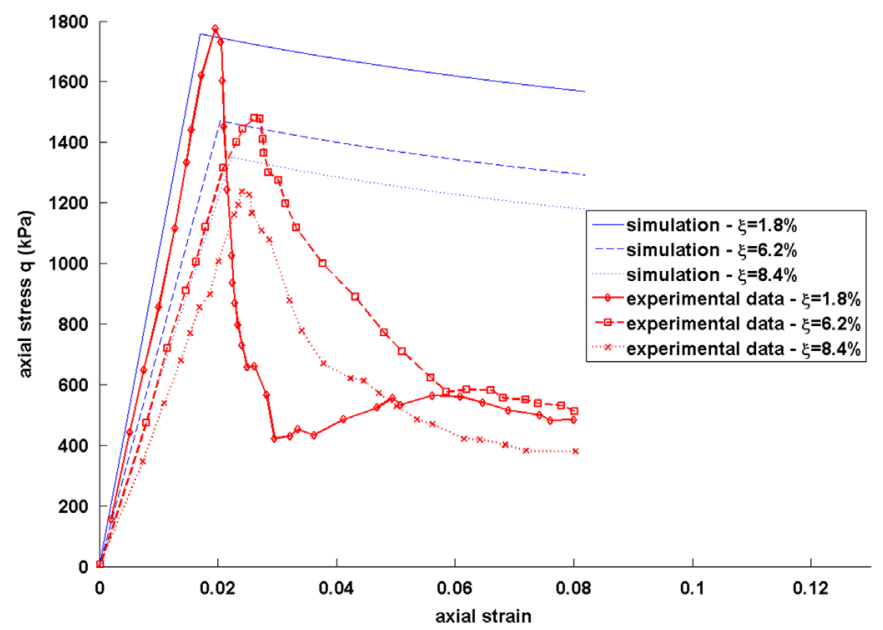

Fig. 6. Comparison of simulations and experimental data ${ }^{31}$ in terms of deviatoric (axial) stress versus axial strain in unconfined compression tests on calcarenite, carried out after weathering up to three different values of degree of dissolution $\xi$.

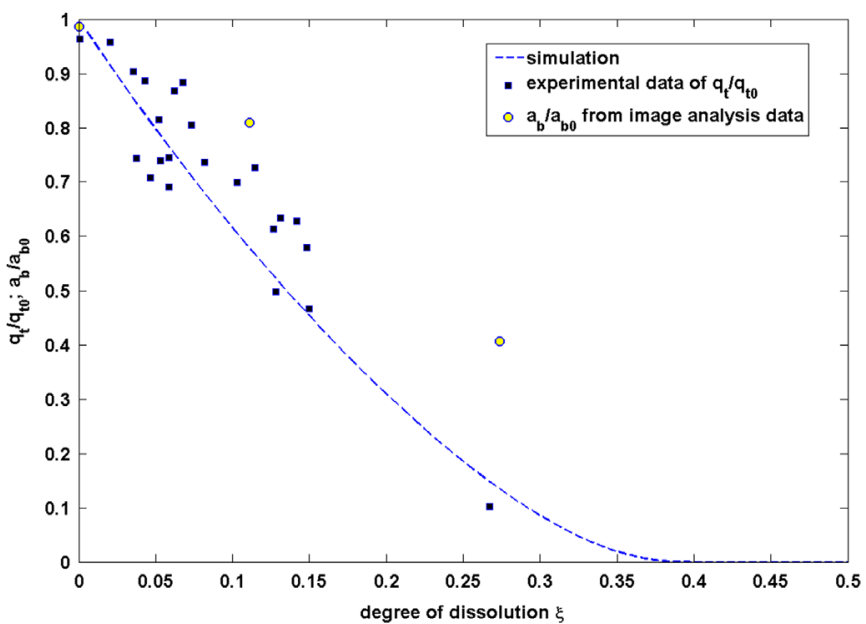

Fig. 7. Comparison of simulations and experimental data in terms of normalized area of bonds $\left(a_{b} / a_{b o}\right)$, or equivalently normalized tensile strength $\left(q_{t} / q_{t o}\right)$, versus the degree of dissolution $\xi$. The experimental data are taken from Ciantia et al.'s ${ }^{31}$ Brazilian test results.

deforming sample (cf. ${ }^{52,53}$ ), additional ad-hoc constitutive assumptions were not introduced for more accurate reproduction of this phenomenon.

More details of the acid weathering phase, that precedes mechanical loading, are simulated in Fig. 7, where the ratio of the current specific cross section area of bonds over its initial value before weathering, $a_{b} / a_{b 0}$, is plotted versus $\xi$. Since from Eq. (2) both material's compressive and tensile strength evolve with the specific cross-section area of bonds, the same curve also represents the simulated evolution with chemical dissolution/deposition of ratios $p_{\text {tens }} / p_{\text {tens } 0}$ and $p_{\text {comp }} / p_{\text {comp } 0}$. In the same plot, both experimental data of normalized tensile strength $\left(q_{t} / q_{t o}\right)$ measured with Brazilian tests, ${ }^{31}$ and the data points of $a_{b} / a_{b 0}$ obtained by Ciantia et al. ${ }^{31}$ by means of image processing of thin sections of calcarenite, are reported, for comparison with the simulated data. The tensile strength data exhibit a very similar trend to simulations. In contrast, despite the experimentally observed trends of variation with $\xi$ of both tensile and compressive strength being curved, the normalized cross sectional bond area derived from image processing seems to decrease linearly with $\xi$, hence the simulations cannot reproduce them accurately. However, both the numerical and the experimental data, respectively with a curved and a linear trend, lead to identify the 'critical' value of $\xi$ corresponding to the vanishing of bond cross-sectional areas to about 0.4-0.5.

In Figs. 8 and 9 the simulated normalized uniaxial compressive strength $q_{c} / q_{c o}$ (i.e. ratio of weathered to initial UCS, obtained by extracting peak stress values from several uniaxial compression simulations in the range $\xi=0-0.35)$ and the normalized elastic modulus $E / E_{0}$ respectively are plotted versus $\xi$, and compared to the corresponding experimental data, showing satisfactory agreement in both cases.

Further, the parameter set \#1 of Table 1 was also used to simulate oedometer tests on calcarenite samples previously subjected to different degrees of weathering. In Fig. 10, satisfactory simulations are shown in terms of vertical strain versus vertical stress, at different degrees of dissolution. Experimental data from ${ }^{31}$ are also reported in the graph for comparison.

\subsubsection{Chemo-mechanical oedometer tests}

A second, different set of chemo-mechanical simulations was performed to reproduce the special oedometer tests, allowing measurement of radial stress, carried out on calcarenite by 


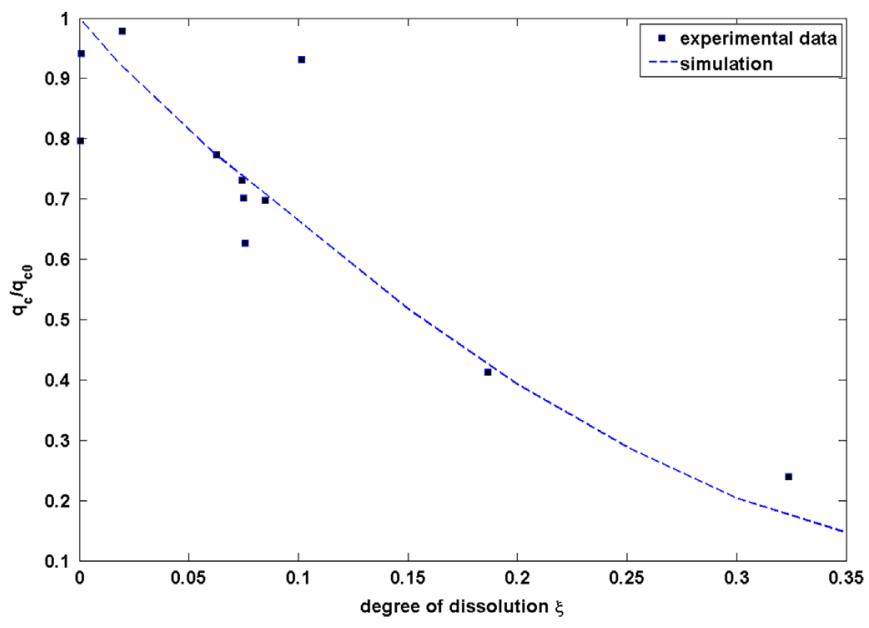

Fig. 8. Comparison of simulations and experimental data in terms of normalized UCS versus the degree of dissolution. The experimental data are taken from Ciantia et al.'s ${ }^{31}$ uniaxial compression test results.

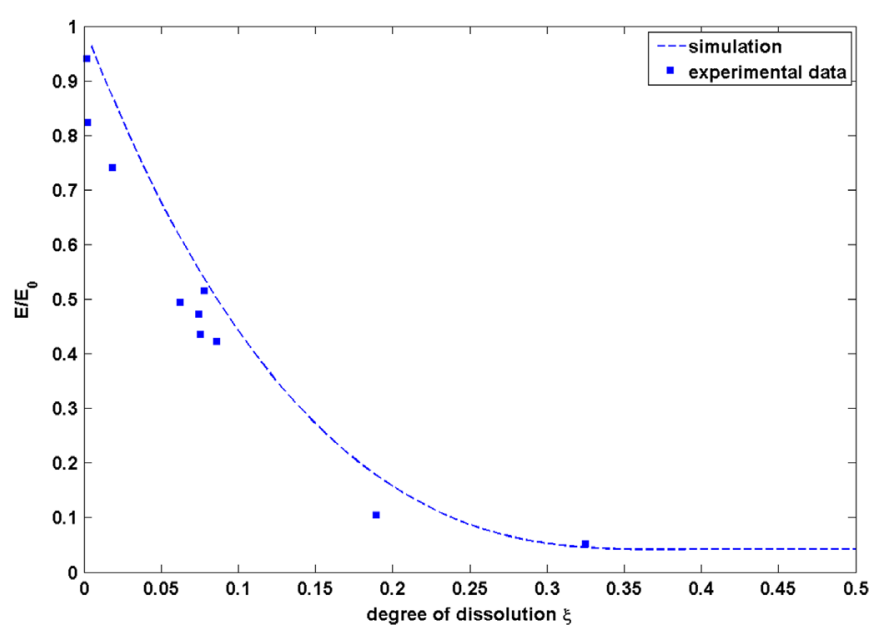

Fig. 9. Comparison of simulations and experimental data in terms of normalized elastic modulus versus the degree of dissolution. The experimental data are taken from Ciantia et al.'s $\mathrm{s}^{31}$ uniaxial compression test results.

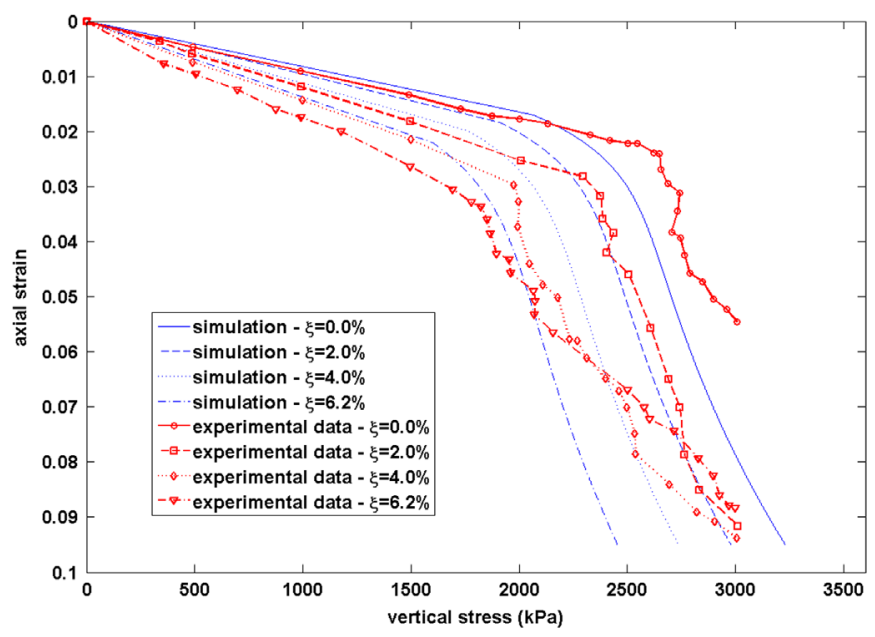

Fig. 10. Comparison of simulations and experimental data ${ }^{31}$ in terms of axial strain versus vertical stress in oedometer tests on calcarenite, carried out after weathering up to four different values of degree of dissolution.

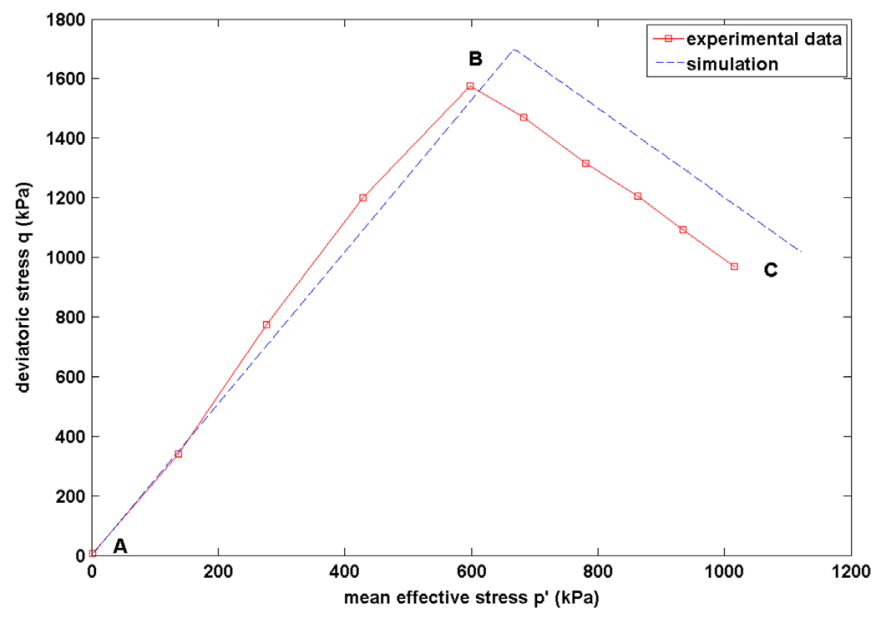

Fig. 11. Comparison of simulations and experimental data ${ }^{30}$ in terms of deviatoric stress versus mean effective stress in an oedometer test on calcarenite. Path A-B represents the mechanical loading phase in unweathered conditions, path $\mathrm{B}-\mathrm{C}$ represents the acid weathering phase at constant applied load.

Castellanza and Nova, ${ }^{30}$ in which the material was subject to acid weathering after the application of a constant vertical load. For consistency, presuming that the tested calcarenite belongs to the same formation as that tested by Ciantia et al., ${ }^{31}$ the same parameter set \#1 of Table 1 was adopted, despite the different experimental conditions.

In Fig. 11 the stress path of Castellanza and Nova's ${ }^{30}$ oedometer test in the $(p, q)$ plane is simulated, and fittingly compared to experimental data. The path $\mathrm{A}-\mathrm{B}$ represents the mechanical loading phase. Upon reaching point $B$, the vertical load is kept constant and the acid weathering phase starts, gradually causing an increase of radial stress, revealing as a linear decrease of deviatoric stress with increasing mean stress (path B-C).

In Fig. 12a the simulated evolution of radial stress during the weathering phase of the test is shown as a function of $\xi$. Similar experimental data are plotted in the same figure for comparison, however it should be noted that the two plots are not exactly equivalent, since the experimental data are reported as a function of time since the start of weathering (Fig. 11 of Castellanza and $\mathrm{Nova}^{30}$ ), and no information is available to deduce the degree of dissolution $\xi$. The initial horizontal part of the simulation represents the chemo-elastic phase at constant applied stress, at the end of which the shrinking yield locus attains the stress point, and chemo-plastic loading starts. Along the same lines, in Fig. 12b the simulated axial strain during the weathering phase of the test is shown as a function of $\xi$, together with the experimental measurements of axial strain as a function of time, for approximate comparison. To better explain these simulation trends, in Fig. 13a the simulated evolution of preconsolidation pressure $p_{c}$ versus degree of dissolution is shown. It can be noted that only elastic loading occurs up to point D in the graph (also marked in Fig. 12a), followed by plastic loading: an initial softening phase implies dilative volumetric behavior, until point $\mathrm{E}$ is reached (also marked in Fig. 12b), representing a transition between dilative and contractive volumetric behavior. This point implies for the stress path the passage by the yield locus' horizontal tangent point, where the plastic volumetric strain rate is zero while the size of the yield locus continues to evolve (cf. Section 3.1). The evolution of ratio $a_{b} / a_{b 0}$ during the weathering phase is shown in Fig. 13b against mean effective stress. It can be noticed that initially, in elastic conditions, the mean stress does not change with dissolution, and starts increasing only upon reaching plastic loading conditions. 

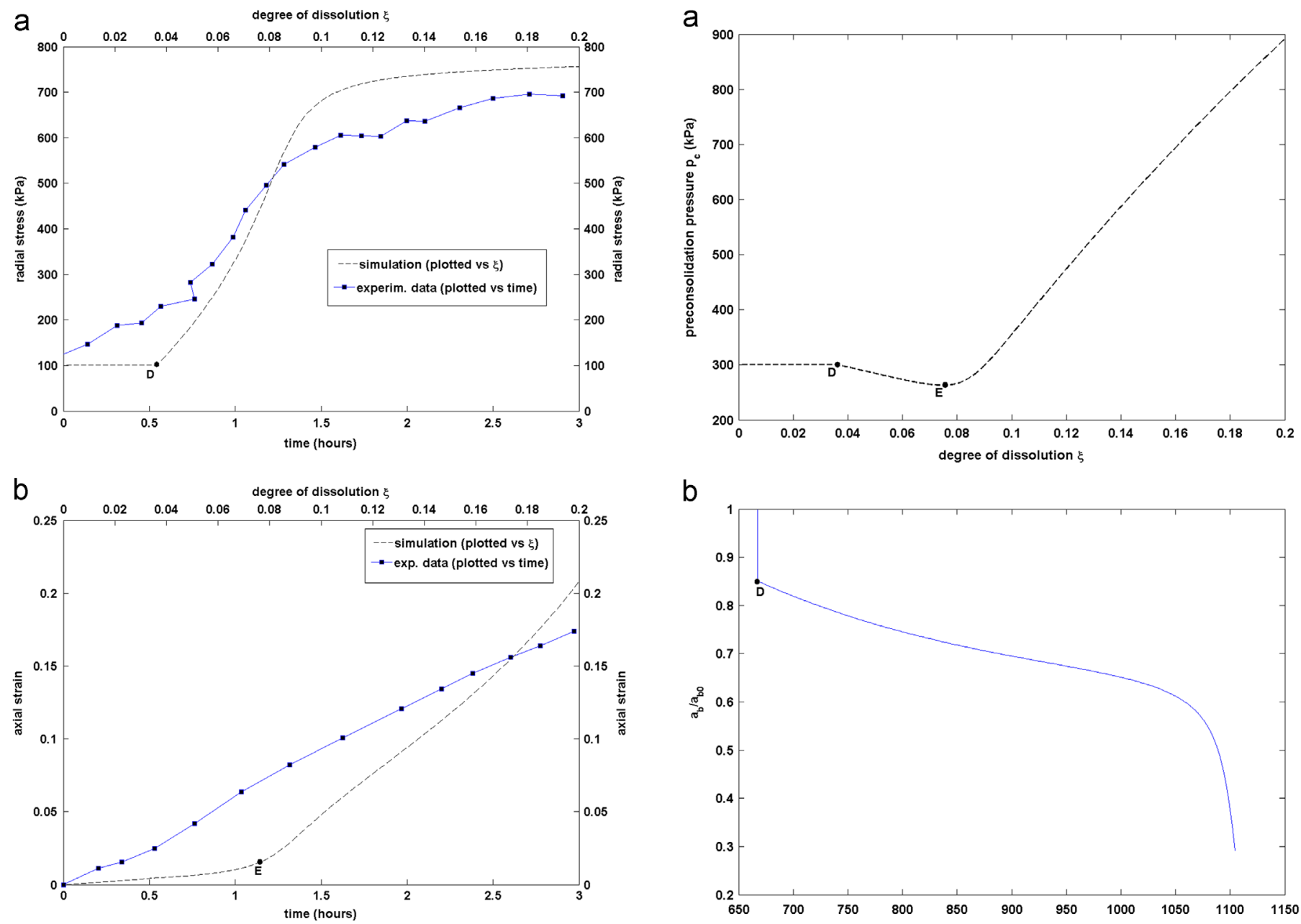

b

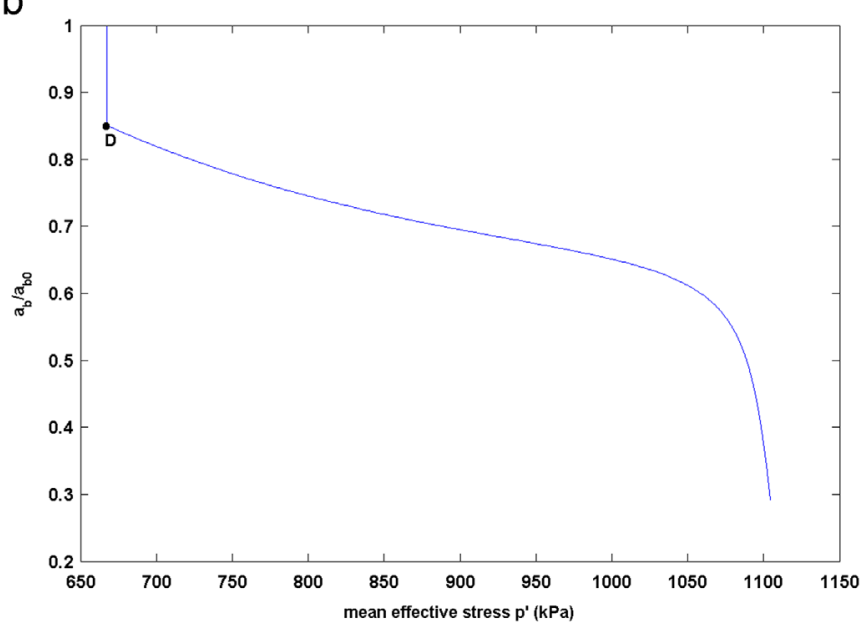

Fig. 12. Comparison of simulations and experimental data ${ }^{30}$ in terms of (a) radial stress evolution and (b) axial strain evolution, during the weathering phase at constant applied load in oedometric conditions. The two datasets are not entirely comparable, since simulated values are plotted against the degree of dissolution (upper horizontal axis), while the experimental data are reported as a function of time (lower horizontal axis).

\subsection{Purely mechanical axisymmetric tests on bonded materials}

The model was further tested by reproducing mechanical tests on bonded materials, without invoking chemical couplings. In this case, reference is made to the experimental evidence gathered by Lagioia and Nova, ${ }^{36}$ who presented a number of axisymmetric tests on a calcarenite. The set of model parameters adopted for these simulations is shown as set \#2 in Table 1. Fig. 14 shows experimental data and the relevant simulation of an isotropic compression test in terms of void ratio variation as a function of mean effective stress. The typical abrupt volumetric collapse at nearly constant stress, indicating destructuration of the material, is effectively reproduced by the model. In Fig. 15 the comparison between the simulations and experimental results of a drained triaxial compression test at a constant cell pressure of $900 \mathrm{kPa}$ is shown both in terms of deviatoric stress and of volumetric strain versus axial strain. While the stress-strain trend is correctly captured by the model, the numerical reproduction of experimental results in terms of volumetric versus axial strain is not as accurate, but still of comparable order of magnitude. The main source of inconsistency is related to the impossibility of simulating exactly

Fig. 13. Simulated evolution of (a) preconsolidation pressure $\mathrm{p}_{\mathrm{c}}$ versus degree of dissolution, and (b) normalized cross-sectional area of bonds against mean effective stress, during the weathering phase at constant applied load in oedometric conditions. It should be noted that point $\mathrm{D}$ marks the onset of plastic yield (cf Fig. 12a), followed by an initial softening phase implying dilative behavior. Point $\mathrm{E}$ represents a transition between dilative and contractive volumetric behavior (cf. Fig. 12b), corresponding to zero plastic volumetric strain rate.

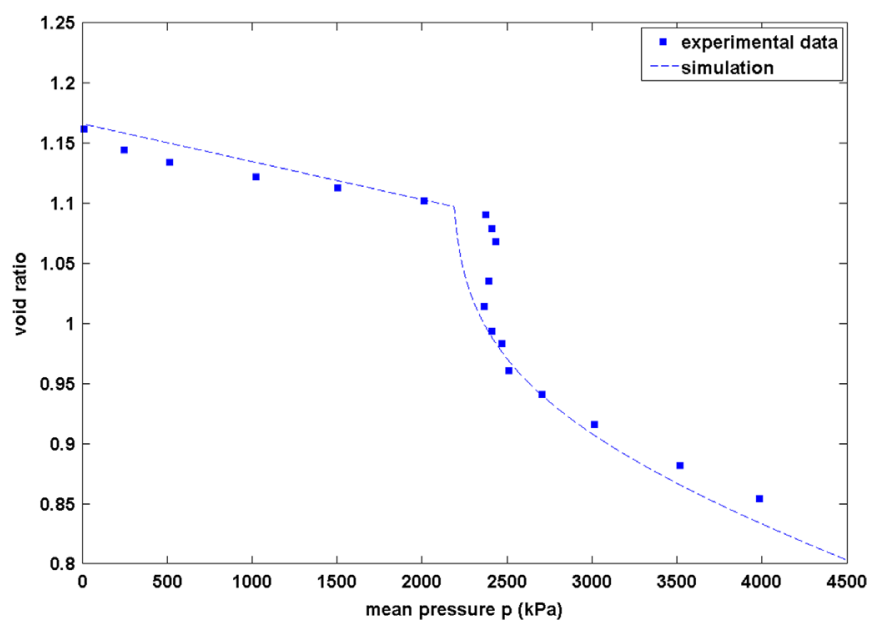

Fig. 14. Comparison of simulations and experimental data ${ }^{36}$ in terms of axial strain versus axial stress in a purely mechanical isotropic compression test on unweathered calcarenite. 


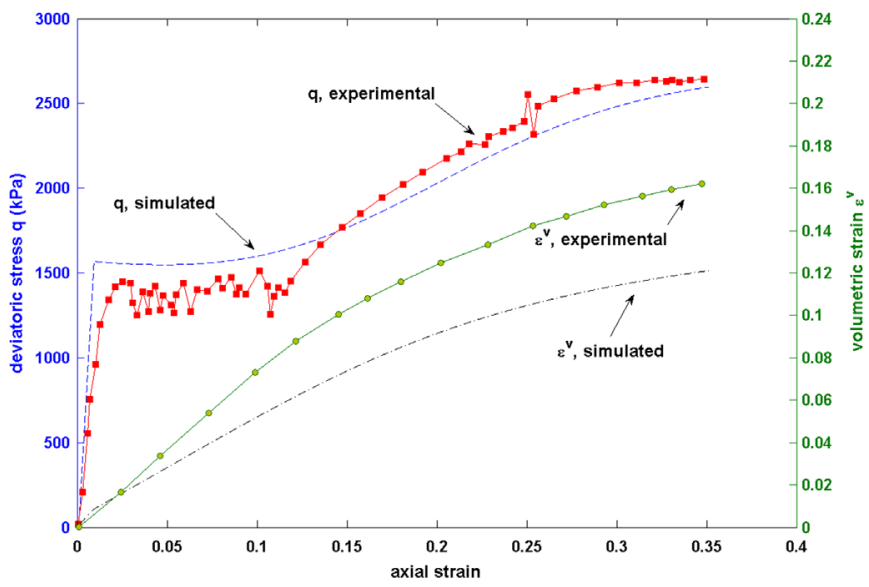

Fig. 15. Comparison of simulations and experimental data [36] in terms of deviatoric stress, and volumetric strain, versus axial strain, in a purely mechanical drained triaxial compression test on unweathered calcarenite.

the yield stresses in isotropic compression and triaxial compression. This inconsistency is related to the assumed shape of the yield surface, thus the simulations could be improved by substituting the yield surface of Cam Clay model with a yield surface having a less rounded shape in the axes origin (e.g., ${ }^{56,57}$ ).

\section{Conclusion}

In this work an innovative, general two-scale modeling framework able to account for the key aspects of the coupled chemomechanical behavior of bonded granular materials is presented, and validated against experimental evidence. The proposed framework includes: an integrated model for mass change of minerals, in the frame of an idealized evolving micro-scale structure of grains and bonds, subject to removal or addition of mineral mass, localized mechanical failure and chemical healing, and a macro-scale chemo-elasto-plastic model, where both the yield stress under isotropic compression and the isotropic tensile strength depend on the material microstructural features, incorporating elasto-plastic coupling to describe the degradation of elastic properties due to mechanically induced debonding.

The key feature of the proposed model is the introduction of two variables: the (total) specific reactive surface area and the mean cross sectional area of the bonds. The specific reactive surface area has the key role of governing the rate of chemical dissolution/precipitation (cementation) and depends on the current amount of cement (micro-fracturing induced by plastic strains is neglected in a first approximation). The mean cross sectional area of the bonds defines the increase/decrease of strength and stiffness due to cementation bonds and depends on bond breakage and on the amount of dissolution/cementation. The specific reactive surface area and the mean cross section area of the bonds are deduced from a micromechanical model, with the advantage of avoiding unphysical situations in which deposition could occur with no porous space available or dissolution could occur with no cementing material left.

In addition, the proposed framework permits to keep track of the evolution of important micromechanical quantities such as the number of active (i.e. non-broken) bonds and their cross sectional area. For the sake of simplicity, the distribution of bonds and their cross sections are assumed isotropic and the model presentation is based on simple linear isotropic elasticity and Cam Clay yield function. The proposed constitutive framework is not however limited to these simple assumptions and could be straightforwardly applied to more complex elasticity models and yield functions. Moreover, the proposed framework could be easily extended to situations involving the presence of different families of cementation bonds characterized by different strengths and chemical dissolution properties, such as those experimentally observed by Ciantia and Hueckel $^{5}$ and Ciantia et al. ${ }^{31}$ on calcarenite.

It can be concluded that the presented model provides a powerful and flexible framework for adequately reproducing different experimental chemo-mechanical loading paths. Due to limited availability of experimental results in the literature, only a few examples focused on the behavior of calcarenite are presented here. However, it is worth remarking that the model is to be intended as an open framework rather than being specialized on a particular material type. Different additional effects, such as mechanical anisotropy, could easily be incorporated depending on modeling needs.

\section{Acknowledgments}

The first two authors gratefully acknowledge financial support from European Union FP7 project under contract number PIAPPGA-2013-609758-HOTBRICKS.

The third author gratefully acknowledges financial support from the "Visiting Professor 2013" program of the University of Trento.

Dr. Matteo Ciantia of UPC and Prof. Riccardo Castellanza of the University of Milan, Bicocca are acknowledged for kindly providing some of their data for our calibrations.

\section{References}

1. Terzaghi K. Mechanism of Landslides. Cambridge: Harvard University, Department of Engineering; 1950.

2. Moore R. The chemical and mineralogical controls upon the residual strength of pure and natural clays. Geotechnique. 1996;41(1)35-47.

3. Moore R, Brunsden D. Physico-chemical effects on the behaviour of a coastal mudslide. Geotechnique. 1996:46(2)259-278.

4. Chigira M, Oyama T. Mechanism and effect of chemical weathering of sedimentary rocks. Eng Geol. 2000;55:3-14.

5. Ciantia MO, Hueckel T. Weathering of submerged stressed calcarenites: chemomechanical coupling mechanisms. Geotechnique. 2013;63(9)768-785.

6. Hueckel T, Hu LB. Feedback mechanisms in chemo-mechanical multi-scale modeling of soil and sediment compaction. Comput Geotech. 2009;36:934-943.

7. Castellanza R. Nova R. Gerolymatou E. An attempt to predict the failure time of abandoned mine pillars. Rock Mech Rock Eng. 2008;41(3)377-401.

8. Zhao Y, Cui P, Hu LB, Hueckel T. Multi-scale chemo-mechanical analysis of the slip surface of landslides in the Three Gorges, China. Sci China Technol Sci. 2011:54:1757-1765.

9. Andre L, Azaroual M, Menjoz A. Numerical simulations of the thermal impact of supercritical $\mathrm{CO}_{2}$ injection on chemical reactivity in a carbonate saline reservoir Transp Porous Media. 2010;82(1)247-274.

10. Nguyen MT, Bemer E, Dormieux L. Micromechanical modeling of carbonate geomechanical properties evolution during acid gas injection. In: Proceedings of the 45th US Rock Mechanics/Geomechanics Symposium. San Francisco, CA, June 26-29, 2011.

11. Hu LB, Hueckel T. Coupled chemo-mechanics in evolving permeability in geomaterials. In: Proceedings of the Computational Geomechanics (ComGeo II). Cavtat-Dubrovnik; 2011. p. 599-608.

12. Kahlweit M. Ostwald ripening of precipitates. Adv Colloid Interface Sci. 1975;5 (1) 135.

13. Sjoberg EL. A fundamental equation for calcite dissolution kinetics. Geochim Cosmochim Acta. 1976;40:441-447.

14. Rimstidt JD, Barnes DL. The kinetics of silica-water reaction. Geochim Cosmochim Acta. 1980;44:1683-1699.

15. Hu LB, Hueckel T. Coupled chemo-mechanics of intergranular contacts: toward a three-scale model. Comput Geotech. 2007;34(4)306-327.

17. Hueckel T. Reactive plasticity for clays during dehydration and rehydration. Part I: Concepts and options. Int J Plast. 2002;18(3)281-312.

18 Loret B, Hueckel T, Gajo A. Chemo-mechanical coupling in saturated porous media: elasto-plastic behaviour of homoionic expansive clays. Int J Solids Struct. 2002;39:2773-2806.

19. Gens A, Nova R. Conceptual bases for a constitutive model for bonded soils and 
weak rocks. In: Proceedings of the International Symposium on Geotechnical Engineering of Hard Soils - Soft Rocks. Athens, Greece; 1993.

20. Nova R. Modelling the weathering effects on the mechanical behaviour of granite. In: Kolymbas D, editor. Constitutive Modelling of Granular Materials. Berlin: Springer; 2000. p. 397-411.

21. Nova R, Castellanza R, Tamagnini C. A constitutive model for bonded geomaterials subject to mechanical and/or chemical degradation. Int J Numer Anal Methods Geomech. 2003;27(9)705-732.

22. Matsukura Y, Hirose T. Five year measurements of rock tablet weathering on a forested hillslope in a humid temperate region. Eng Geol. 1999;55:69-76.

23. Kohler SJ, Dufaud F, Oelkers EH. An experimental study of illite dissolution kinetics as a function of $\mathrm{pH}$ from 1.4 to 12.4 and temperature from 5 to $50{ }^{\circ} \mathrm{C}$. Geochim Cosmochim Acta. 2002;67(19)3583-3594.

24. Hueckel T. Coupling of elastic and plastic deformations of bulk solids. Meccanica. 1976;11(4)227-235.

25. Maier G, Hueckel T. Nonassociated and coupled flow rules of elastoplasticity for rock-like material. Int J Rock Mech Min Sci Geomech Abstr. 1979;16:77-92.

26. Hueckel T. Discretized kinematic hardening in cyclic degradation of rocks and soils. Eng Fract Mech. 1985;21(4)923-945.

27. Hueckel T, Tutumluer E. Modeling of elastic anisotropy due to one-dimensional plastic consolidation of clays. Comput Geotech. 1994;16:311-349.

28. Gajo A, Bigoni D. A model for stress and plastic strain induced nonlinear, hyperelastic anisotropy in soils. Int J Numer Anal Methods Geomech. 2008;32(7) 833-861.

29. Gajo A. Hyperelastic modelling of small-strain stiffness anisotropy of cyclically loaded sand. Int J Numer Anal Methods Geomech. 2010;34(2)111-134.

30 Castellanza R, Nova R. Oedometric tests on artificially weathered carbonatic soft rocks. J Geotech Geoenviron Eng ASCE. 2004;130(7)28-739.

31. Ciantia MO, Castellanza R, di Prisco C. Experimental study on the water-induced weakening of calcarenites. Rock Mech Rock Eng. 2014, http://dx.doi.org/10.1007/ s00603-014-0603-Z.

36. Lagioia R, Nova R. An experimental and theoretical study of the behaviour of a calcarenite in triaxial compression. Geotechnique. 1995;45(4)633-648.

37. Gajo A, Muir Wood D. A new approach to anisotropic, bounding surface plasticity: general formulation and simulations of natural and reconstituted clays. Int J Numer Anal Methods Geomech. 2001;25:207-241.

38. Yu HS, Khong CD, Wang J, Zhang G. Experimental evaluation and extension of a simple critical state model for sand. Granul Matter. 2005;7:213-225.
39. Yan WM, Li XS. A model for natural soils with bonds. Geotechnique. 2011;61(2 95-106.

40. Hueckel T. Water-mineral interaction in hygro-mechanics of clays exposed to environmental loads: a mixture theory approach. Can Geotech J. 1992;29:10711108.

41. Gajo A, Loret B, Hueckel T. Electro-chemo-mechanical couplings and the elastic-plastic behaviour of heteroionic expansive clays. In: Di Maio C, Hueckel T, Loret B, editors. Chemo-mechanical Coupling in Clays. Rotterdam: Balkema; 2002. p. 261-276.

42. Muir Wood D. Soil Behaviour and Critical State Soil Mechanics. New York: Cambridge University Press; 1990.

43. Roscoe KH, Burland JB. On the generalised stress-strain behaviour of 'wet' clay. Eng Plastic. 1968:535-609.

44. Willam KJ, Warnke EP. Constitutive models for the triaxial behavior of concrete. In: Proceedings of the International Association for Bridge and Structural Engineering; 1975. 19: 1- 30.

45. Desai CS, Siriwardane HJ. Constitutive Laws for Engineering Materials. Englewood Cliffs: Prentice-Hall; 1984.

48. Haase R. Thermodynamics of Irreversible Processes. New York: Dover Publications; 1990.

49. Brantley S, Kubicki J, Art W. Kinetics of Rock-Water Interaction. New York: Springer; 2007.

50. Murphy WF. Effect of Microstructure and Pore Fluids on the Acoustic Properties of Granular Sedimentary Materials [Ph.D. thesis]. USA: Stanford University; 1982.

52. Gajo A, Bigoni D, Muir Wood D. Multiple shear band development and related instabilities in granular materials. J Mech Phys Solids. 2004;52(12)2683-2724.

53. Gajo A, Muir Wood D, Bigoni D. On certain critical material and testing characteristics affecting shear band development in sand. Geotechnique. 2007;57(5) 449-461.

54. Ou C, Wu T, Hsieh H. Analysis of deep excavation with column type of ground improvement in soft clay. J Geotech Eng. 1996;122(9)709-716.

55. Goodman RE. Introduction to Rock Mechanics. Chichester: John Wiley \& Sons; 1989

56. Nova R, Hueckel T. A unified approach to the modelling of liquefaction and cyclic mobility of sands. Soils Found. 1981;21(4)13-28.

57. Bigoni D, Piccolroaz A. Yield criteria for quasibrittle and frictional materials. Int ] Solids Struct. 2004:41(11-12)2855-2878. 